Pacific

Journal of

Mathematics

CLOSED CONFORMAL VECTOR FIELDS AND

LAGRANGIAN SUBMANIFOLDS IN COMPLEX SPACE FORMS

Ildefonso Castro, Cristina R. Montealegre, and Francisco

URBANO 


\title{
CLOSED CONFORMAL VECTOR FIELDS AND LAGRANGIAN SUBMANIFOLDS IN COMPLEX SPACE FORMS
}

\author{
Ildefonso Castro, Cristina R. Montealegre, and Francisco \\ URBANO
}

We study a wide family of Lagrangian submanifolds in nonflat complex space forms that we will call pseudoumbilical because of their geometric properties. They are determined by admitting a closed and conformal vector field $X$ such that $X$ is a principal direction of the shape operator $A_{J X}$, being $J$ the complex structure of the ambient manifold. We emphasize the case $X=J H$, where $H$ is the mean curvature vector of the immersion, which are known as Lagrangian submanifolds with conformal Maslov form. In this family we offer different global characterizations of the Whitney spheres in the complex projective and hyperbolic spaces.

Let $\bar{M}^{n}$ be a Kaehler manifold of complex dimension $n$. The Kaehler form $\Omega$ on $\bar{M}$ is given by $\Omega(v, w)=\langle v, J w\rangle$, being $\langle$,$\rangle the metric and J$ the complex structure on $\bar{M}$. An immersion $\phi: M \longrightarrow \bar{M}$ of an $n$-dimensional manifold $M$ is called Lagrangian if $\phi^{*} \Omega \equiv 0$. This property involves only the symplectic structure of $\bar{M}$. In this family of Lagrangian submanifolds, one can study properties of the submanifold involving the Riemannian structure of $\bar{M}$. One must take into account the nice property of the second fundamental form $\sigma$ of these submanifolds which says that the trilinear form

$$
\langle\sigma(v, w), J z\rangle
$$

is totally symmetric. Sometimes this property of the second fundamental form gives obstructions to the existence of examples satisfying classical Riemannian properties. So, if one considers like classical property the umbilicity, automatically our umbilical Lagrangian submanifold is totally geodesic and only appear trivial examples. Then two natural questions arise: What is the Lagrangian version of umbilicity? Which are the corresponding examples?.

In $[\mathbf{R U}]$ it was proposed that the Lagrangian version of umbilicity was that the second fundamental form satisfies

$$
\langle\sigma(v, w), J z\rangle=\frac{n}{n+2} \partial_{v, w, z}\langle v, w\rangle\langle H, J z\rangle,
$$


where the symbol $\partial_{v, w, z}$ means cyclic sum over $v, w, z$ and $H$ is the mean curvature vector of the submanifold. The corresponding classification was also made when the ambient space was complex Euclidean space $\mathbb{C}^{n}$. Besides the linear Lagrangian subspaces, the Whitney spheres ([RU], see Paragraph 4 for the definition) were the only examples, which can be considered like the Lagrangian version of the round hyperspheres of Euclidean space. For $n=2$ this classification was made in [CU1].

In [CU2] when $n=2$ and in [Ch1] in arbitrary dimension, the Lagrangian submanifolds satisfying (0.1) of the complex projective and hyperbolic spaces $\mathbb{C P}^{n}$ and $\mathbb{C H}^{n}$ were classified. Again, in the complex projective space only the Whitney spheres appeared, but in the complex hyperbolic space, besides the Whitney spheres, two new families of noncompact examples appeared, topologically equivalent to $\mathbb{S}^{1} \times \mathbb{R}^{n-1}$ and $\mathbb{R}^{n}$, which can be considered like the Lagrangian version of the tubes over hyperplanes and the horospheres in the real hyperbolic space.

Following with the analogy between umbilical hypersurfaces of real space forms and our family of Lagrangian submanifolds of complex space forms, the umbilical hypersurfaces are the easiest examples of submanifolds with constant mean curvature. Our Lagrangian examples, except the totally geodesic ones, do not have parallel mean curvature vector, property which is usually taken as a version on higher codimension of the notion of constant mean curvature, but their mean curvature vectors $H$ satisfy that $J H$ are conformal fields on the submanifolds. So, we will take this property like the Lagrangian version of the concept of hypersurfaces of constant mean curvature. As the dual form of $J H$ is the well-known Maslov form, we will refer to these submanifolds as Lagrangian submanifolds with conformal Maslov form. In [RU] this family was studied when the ambient space is $\mathbb{C}^{n}$.

The Lagrangian submanifolds satisfying (0.1) also verify that $J H$ is a principal direction of $A_{H}$. Motivated by this fact, in this paper we study a wide family of Lagrangian submanifolds, defined by the property that the submanifold admits a closed and conformal vector field $X(J H$ is always a closed field) such that $X$ is a principal direction of $A_{J X}$, without assuming that $J X$ is the mean curvature vector $H$. In this family a minor important degenerate case appears, which is completely studied and classified in Paragraph 3. Motivated by Proposition 1, the nondegenerate submanifolds of our family are called pseudoumbilical. A particular family of this kind of submanifolds, which was called H-umbilical by B.Y. Chen, was studied in [Ch2].

In Paragraphs 1 and 2 we study deeply pseudoumbilical Lagrangian submanifolds of $\mathbb{C P}^{n}$ and $\mathbb{C H}^{n}$. In Theorem 1, we describe the pseudoumbilical Lagrangian submanifolds (see Definition 1), showing that they have a similar behavior to the "umbilical" Lagrangian submanifolds. Theorem 1 allows 
us to classify pseudoumbilical Lagrangian submanifolds of $\mathbb{C P}^{n}$ and $\mathbb{C H} \mathbb{H}^{n}$ (Corollary 1 and Theorem 2); they are described in terms of planar curves and Lagrangian (n-1)-submanifolds of the complex projective, hyperbolic or Euclidean spaces depending on the elliptic, hyperbolic or parabolic character of the submanifold.

In Paragraph 4, we use the above classification in order to study Lagrangian submanifolds with conformal Maslov form. Among the most important global results, we show the following Lagrangian version of a Hopf theorem:

The Whitney spheres are the only compact (nonminimal) Lagrangian submanifolds of $\mathbb{C P}^{n}$ and $\mathbb{C} \mathbb{H}^{n}$ with conformal Maslov form and null first Betti number.

Another global result is established in terms of the Ricci curvature. In this context, we prove in Corollary 3 that the Whitney spheres are the only compact (nonminimal) Lagrangian submanifolds of $\mathbb{C P}^{n}$ with conformal Maslov form such that $\operatorname{Ric}(J H) \geq(n-1)|H|^{2}$.

Finally, we prove that all the orientable compact Lagrangian submanifolds of $\mathbb{C P}^{n}$ and $\mathbb{C H}^{n}$ with nonparallel conformal Maslov form and first Betti number one are elliptic pseudoumbilical and then this allows describe them (Corollary 4).

\section{Closed conformal fields and Lagrangian submanifolds.}

The Lagrangian submanifolds we are going to consider in this paper will have a closed and conformal vector field. So, in this section, we will describe properties of this kind of vector fields, as well as properties of Lagrangian submanifolds admitting this kind of vector fields.

The following lemma summerizes some of the known results about Riemannian manifolds which admit closed and conformal vector fields (see [RU] and references there in).

Lemma 1. Let $(M,\langle\rangle$,$) be an n$-dimensional Riemannian manifold endowed with a nontrivial vector field $X$ which is closed and conformal. Then:

(i) The set $\mathcal{Z}(X)$ of the zeros of $X$ is a discrete set.

(ii) If $\operatorname{div} X$ denotes the divergence of $X$, then

$$
\nabla_{V} X=\frac{\operatorname{div} X}{n} V, \quad|X|^{2} \nabla(\operatorname{div} X)=-\frac{n \operatorname{Ric}(X)}{n-1} X,
$$

for any vector field $V$ on $M$, where Ric denotes the Ricci curvature of $M$.

(iii) The curvature tensor $R$ of $M$ satisfies

$$
|X|^{2} R(v, w) X=\frac{\operatorname{Ric}(X)}{n-1}\{\langle w, X\rangle v-\langle v, X\rangle w\}
$$


(iv) If $M^{\prime}=M-\mathcal{Z}(X)$, then

$$
p \in M^{\prime} \mapsto \mathcal{D}(p)=\left\{v \in T_{p} M /\langle v, X\rangle=0\right\}
$$

defines an umbilical foliation on $\left(M^{\prime},\langle\rangle,\right)$. In particular, $|X|^{2}$ and $\operatorname{div} X$ are constant on the connected leaves of $\mathcal{D}$.

(v) $\left(M^{\prime}, g\right)$ with $g=|X|^{-2}\langle$,$\rangle , is locally isometric to \left(I \times N, d t^{2} \times g^{\prime}\right)$, where $I$ is an open interval in $\mathbb{R},\{t\} \times N$ is a leaf of the foliation $\mathcal{D}$ for any $t \in \mathbb{R}$, and $X=(\partial / \partial t, 0)$. Moreover, if $\Delta^{g}$ is the Laplacian of $g$ then

$$
\Delta^{g} \log |X|+\frac{\operatorname{Ric}(X)}{n-1}=0 .
$$

In this paper, $\bar{M}^{n}(c)$ will denote a complete simply-connected complex space form with constant holomorphic sectional curvature c, with $c=4,0,-4$, i.e., complex Euclidean space $\mathbb{C}^{n}$ if $c=0$, the complex projective space $\mathbb{C P}^{n}$ if $c=4$ and the complex hyperbolic space $\mathbb{C H}^{n}$ if $c=-4$.

Let $\mathbb{S}^{2 n+1}=\left\{z \in \mathbb{C}^{n+1}:\langle z, z\rangle=1\right\}$ be the hypersphere of $\mathbb{C}^{n+1}$ centered at the origin with radius 1 , where $\langle$,$\rangle denotes the inner product on \mathbb{C}^{n+1}$. We consider the Hopf fibration $\Pi: \mathbb{S}^{2 n+1} \longrightarrow \mathbb{C P}^{n}$, which is a Riemannian submersion. We can identify $\mathbb{C}^{n}$ with the open subset of $\mathbb{C P}^{n}$ defined by

$$
\left\{\Pi\left(z_{1}, \ldots, z_{n+1}\right) \in \mathbb{C P}^{n}: z_{n+1} \neq 0\right\} .
$$

Then the Fubini-Study metric $g$ is given on $\mathbb{C}^{n}$ by

$$
g_{p}=\frac{1}{1+|p|^{2}}\left\{\langle,\rangle-\frac{\mathcal{R}(\alpha \otimes \bar{\alpha})}{1+|p|^{2}}\right\}
$$

where $\langle$,$\rangle is the Euclidean metric, \mathcal{R}$ denotes real part and $\alpha$ is the complex 1 -form on $\mathbb{C}^{n}$ given by

$$
\alpha_{p}(v)=\langle v, p\rangle+i\langle v, J p\rangle .
$$

On the other hand, if $c=-4$, let $\mathbb{H}_{1}^{2 n+1}=\left\{z \in \mathbb{C}^{n+1}:(z, z)=-1\right\}$ be the anti-De Sitter space where $($,$) denotes the hermitian form$

$$
(z, w)=\sum_{i=1}^{n} z_{i} \bar{w}_{i}-z_{n+1} \bar{w}_{n+1},
$$

for $z, w \in \mathbb{C}^{n+1}$. Then $\langle z, w\rangle=\mathcal{R}(z, w)$ induces on $\mathbb{H}_{1}^{2 n+1}$ a Lorentzian metric of constant curvature -1 . If $\Pi: \mathbb{H}_{1}^{2 n+1} \longrightarrow \mathbb{C H}^{n}$ denotes the Hopf fibration, it is well-known that $\Pi$ is a Riemannian submersion. Then $\mathbb{C} \mathbb{H}^{n}$ can be identified with the unit ball

$$
\mathbb{B}^{n}=\left\{\left(z_{1}, \ldots, z_{n}\right) \in \mathbb{C}^{n}: \sum_{i=1}^{n}\left|z_{i}\right|^{2}<1\right\},
$$


endowed with the Bergmann metric

$$
g_{p}=\frac{1}{1-|p|^{2}}\left\{\langle,\rangle+\frac{\mathcal{R}(\alpha \otimes \bar{\alpha})}{1-|p|^{2}}\right\} .
$$

We recall that $\mathbb{C H} \mathbb{H}^{n}$ has a smooth compactification $\mathbb{C} \mathbb{H}^{n} \cup \mathbb{S}^{2 n-1}(\infty)$, where $\mathbb{S}^{2 n-1}(\infty)$ can be identified with asymptotic classes of geodesic rays in $\mathbb{C H}^{n}$. It is known that $\mathbb{S}^{2 n-1}(\infty)$ can be also identified with

$$
\mathbb{S}^{2 n-1}(\infty) \equiv \pi(\mathcal{N})
$$

being

$$
\mathcal{N}=\left\{z \in \mathbb{C}^{n+1}-\{0\}:(z, z)=0\right\}
$$

and $\pi$ the projection given by the natural action of $\mathbb{C}^{*}$ over $\mathcal{N}$. This identification is given by

$$
[\beta(s)] \mapsto \pi\left(\widetilde{\beta}(s)+\frac{\widetilde{\beta^{\prime}}(s)}{\left|\widetilde{\beta}^{\prime}(s)\right|}\right)
$$

being $\beta(s), s \geq 0$, a geodesic ray in $\mathbb{C H}^{n}$ and $\widetilde{\beta}(s), s \geq 0$, a geodesic ray in $\mathbb{H}_{1}^{2 n+1}$ with $\Pi(\widetilde{\beta}(s))=\beta(s)$.

Finally, in order to understand Theorem 1, it is convenient to remember that although in $\mathbb{C P}^{n}$ and $\mathbb{C H} \mathbb{H}^{n}$ there do not exist umbilical real hypersurfaces, M. Kon in $[\mathbf{K}]$ and S. Montiel in $[\mathbf{M}]$ proved that the geodesic spheres of $\mathbb{C P}^{n}$ and the geodesic spheres, the tubes over complex hyperplanes and the horospheres of $\mathbb{C H}{ }^{n}$ are the only $\eta$-umbilic real hypersurfaces of $\mathbb{C P}^{n}$ and $\mathbb{C H} \mathbb{H}^{n}$. The horospheres of $\mathbb{C} \mathbb{H}^{n}$ with infinity point $C \in \mathbb{S}^{2 n-1}(\infty)$ and radius $\lambda>0$ are defined by

$$
\left\{\Pi(z) \in \mathbb{C H}^{n}:|(z, \widetilde{C})|^{2}=\lambda^{2}\left|\widetilde{C}_{n+1}\right|^{2}\right\}
$$

where $\widetilde{C}=\left(\widetilde{C}_{1}, \ldots, \widetilde{C}_{n+1}\right)$ is a point in $\mathcal{N}$ such that $\pi(\widetilde{C})=C$.

Let $\phi$ be an isometric immersion of a Riemannian $n$-manifold $M$ in $\bar{M}^{n}(c)$. If the almost complex structure $J$ of $\bar{M}^{n}(c)$ carries each tangent space of $M$ into its corresponding normal space, $\phi$ is called Lagrangian. We denote the Levi-Civita connection of $M$ and the connection on the normal bundle by $\nabla$ and $\nabla^{\perp}$, respectively. The second fundamental form will be denoted by $\sigma$ and the shape operator by $A_{\xi}$. If $\phi$ is Lagrangian, the formulas of Gauss and Weingarten lead to

$$
\begin{aligned}
\nabla_{Y}^{\perp} J Z & =J \nabla_{Y} Z, \\
\sigma(Y, Z)=J A_{J Y} Z & =J A_{J Z} Y,
\end{aligned}
$$

for tangent vector fields $Y$ and $Z$. These formulas imply that $\langle\sigma(Y, Z), J W\rangle$ is totally symmetric, where $\langle$,$\rangle denotes the metric in \bar{M}^{n}(c)$ and the induced one in $M$ by $\phi$. Using the Codazzi equation, $\langle(\nabla \sigma)(Y, Z, W), J U\rangle$ is 
also totally symmetric, where $\nabla \sigma$ is the covariant derivative of the second fundamental form.

If $\phi: M \longrightarrow \mathbb{C} \mathbb{P}^{n}$ is a Lagrangian immersion of a simply-connected manifold $M$, then it is well-known that $\phi$ has a horizontal lift (with respect to the Hopf fibration) to $\mathbb{S}^{2 n+1}$ which is unique up to rotations on $\mathbb{S}^{2 n+1}$. We will denote by $\widetilde{\phi}$ this horizontal lift. The horizontality means that $\left\langle\widetilde{\phi}_{*}(v), J \widetilde{\phi}\right\rangle=0$ for any tangent vector $v$ to $M$, where $J$ is the complex structure of $\mathbb{C}^{n+1}$. We remark that only the Lagrangian immersions in $\mathbb{C P}^{n}$ have (locally) horizontal lifts.

In a similar way, if $\phi: M \longrightarrow \mathbb{C} \mathbb{H}^{n}$ is a Lagrangian immersion of a simplyconnected manifold $M$, then $\phi$ has a horizontal lift to $\mathbb{H}_{1}^{2 n+1}$, that we will denote by $\widetilde{\phi}$. We also remark that only the Lagrangian immersions in $\mathbb{C} \mathbb{H}^{n}$ have (locally) horizontal lifts.

Proposition 1. Let $\phi: M^{n} \longrightarrow \bar{M}^{n}(c)$ be a Lagrangian isometric immersion of a connected Riemannian manifold $M$ endowed with a closed and conformal field $X$. Following the notation of Lemma 1, suppose that $\sigma(X, X)=\rho J X$, for some function $\rho$ defined on $M^{\prime}=M-\mathcal{Z}(X)$. Then $\rho$ is constant on the connected leaves of the foliation $\mathcal{D}$ and we have only two possibilities:

(A) At any point $p$ of $M, A_{J X}$ has two constant eigenvalues $b_{1}$ and $b_{2}$ on $\mathcal{D}(p)$, with constant multiplicities $n_{1}$ and $n_{2}$. In this case, $\rho$ is constant and the field $X$ is parallel.

(B) At any point $p$ of $M^{\prime}, A_{J X}$ has only one eigenvalue $b(p)$ on $\mathcal{D}(p)$. In this case, $b$ is constant on the connected leaves of the foliation $\mathcal{D}$ and

$$
\frac{c}{4}|X|^{2}+\left(\frac{\operatorname{div} X}{n}\right)^{2}+b^{2}=\lambda \in \mathbb{R} .
$$

Remark 1. An immersion $\phi$, under the assumptions of Proposition 1, admits a 1-parameter family of closed and conformal vector fields $X_{\mu}=\mu X$ with $\mu$ a nonnull real number, satisfying $\sigma\left(X_{\mu}, X_{\mu}\right)=\mu \rho J\left(X_{\mu}\right)$. So, if (A) is satisfied, we always will consider one of the two parallel fields $X$ such that $|X|=1$. If condition (B) is satisfied, then $\sigma\left(V, X_{\mu}\right)=\mu b J V$ for $V$ orthogonal to $X_{\mu}$. So

$$
\frac{c}{4}\left|X_{\mu}\right|^{2}+\left(\frac{\operatorname{div} X_{\mu}}{n}\right)^{2}+(\mu b)^{2}=\mu^{2} \lambda .
$$

When $\lambda \neq 0$ we always will consider one of the two closed and conformal fields $X$ such that the corresponding constant $\lambda$ will be 1 or -1 and we will refer to them to be elliptic or hyperbolic cases. The parabolic case will correspond to $\lambda=0$.

Proposition 1 motivates the following definition. 
Definition 1. A Lagrangian immersion $\phi: M^{n} \longrightarrow \bar{M}^{n}(c)$ is said to be pseudoumbilical if the Riemannian manifold $M$ is endowed with a closed and conformal field $X$ (without zeros) such that $\sigma(X, X)=\rho J X$ for certain function $\rho$ on $M$ and condition (B) in Proposition 1 is satisfied.

Remark 2. When $c=4$, a pseudoumbilical Lagrangian immersion is always elliptic. When $c=0$, it can be either parabolic or elliptic. And when $c=-4$, it can be elliptic, parabolic or hyperbolic.

Proof of Proposition 1. We start proving that $\nabla \rho=h X$ for a certain function $h$, which means that $\rho$ is constant on the connected leaves of $\mathcal{D}$. For this purpose, derivating $\sigma(X, X)=\rho J X$ with respect to a tangent vector field $V$ and using Lemma 1, (ii) we get

$$
\begin{array}{r}
\langle(\nabla \sigma)(V, X, X), J W\rangle+\frac{2 \operatorname{div} X}{n}\langle\sigma(V, X), J W\rangle \\
=\langle\nabla \rho, V\rangle\langle X, W\rangle+\frac{\rho \operatorname{div} X}{n}\langle V, W\rangle,
\end{array}
$$

for any field $W$. Using properties of $\phi$ and the Codazzi equation of $\phi$ we obtain that all the terms in the above equation are symmetric on $V$ and $W$, except possible $\langle\nabla \rho, V\rangle\langle X, W\rangle$. So this one must be also symmetric and this means that $\nabla \rho=h X$ for certain function $h$. In particular $\rho$ is constant on the leaves of $\mathcal{D}$.

On the other hand, as $X(p)$ is an eigenvector of $A_{J X}$, then $A_{J X}$ can be diagonalized on $\mathcal{D}(p)$. Now using Lemma 1, (iii) and the Gauss equation, the eigenvalues $\lambda^{\prime} s$ of $A_{J X}$ on $\mathcal{D}(p)$ satisfy the equation

$$
\lambda^{2}-\rho \lambda+\frac{\operatorname{Ric}(X)}{n-1}-\frac{c}{4}|X|^{2}=0 .
$$

In particular there are at most two. From Lemma $1,(\mathrm{v}), \operatorname{Ric}(X)$ is constant on the connected leaves of $\mathcal{D}$. So the two possible eigenvalues of $A_{J X}$ on $\mathcal{D}(p)$ are also constant on the leaves of $\mathcal{D}$.

Let

$$
M_{0}=\left\{p \in M^{\prime} / A_{J X} \text { has two different eigenvalues on } \mathcal{D}(p)\right\} .
$$

Claim. $M_{0}$ is a closed subset of $M^{\prime}$.

If these eigenvalues are denoted by $b_{i}, i=1,2$, and their multiplicities by $n_{i}, i=1,2$, then $n\langle H, J X\rangle=\rho+n_{1} b_{1}+n_{2} b_{2}$. Because $b_{i}$ are smooth functions on $M_{0}$, we have that $n_{i}, i=1,2$ are constant on each connected component of $M_{0}$. So,

$$
\mathcal{D}_{i}(p)=\left\{v \in \mathcal{D}(p) / A_{J X} v=b_{i} v\right\}, i=1,2,
$$

define distributions on the connected components of $M_{0}$ such that $\mathcal{D}=$ $\mathcal{D}_{1} \oplus \mathcal{D}_{2}$. 
If $V_{i}$ are vector fields on $\mathcal{D}_{i}$, from Lemma 1, (iii) and the Gauss equation of $\phi$, we have that

$$
\sigma\left(V_{1}, V_{2}\right)=0 .
$$

Also derivating $\left\langle\sigma\left(V_{i}, V_{i}\right), J X\right\rangle=b_{i}\left\langle V_{i}, V_{i}\right\rangle$ with respect to $V_{j}$, with $j \neq i$, and using (1.3) and the fact that $\nabla b_{i}=h_{i} X$ for certain functions $h_{i}$, we obtain that

$$
\left\langle(\nabla \sigma)\left(V_{j}, V_{i}, V_{i}\right), J X\right\rangle=0,
$$

for $V_{i} \in \mathcal{D}_{i}, V_{j} \in \mathcal{D}_{j}$ and $i \neq j$. But derivating with respect to $V_{i}$, $\left\langle\sigma\left(X, V_{i}\right), J V_{j}\right\rangle=0$ and using (1.3) and (1.4) we get

$$
\left(b_{i}-b_{j}\right)\left\langle\nabla_{V_{i}} V_{i}, V_{j}\right\rangle=0
$$

and then $\left\langle\nabla_{V_{i}} V_{i}, V_{j}\right\rangle=0$ when $i \neq j$. So

$$
\nabla_{V_{i}} V_{i}=-\frac{\operatorname{div} X}{n}|X|^{-2}\left|V_{i}\right|^{2} X+\left(\nabla_{V_{i}} V_{i}\right)^{i}
$$

where ${ }^{i}$ means component on $\mathcal{D}_{i}$. Finally, taking $i \neq j$, derivating $\sigma\left(V_{i}, V_{j}\right)=$ 0 with respect to $V_{i}$ and using the above equation and (1.3) we get

$$
\left\langle(\nabla \sigma)\left(V_{i}, V_{i}, V_{j}\right), J V_{j}\right\rangle=b_{j}(\operatorname{div} X / n)|X|^{-2}\left|V_{i}\right|^{2}\left|V_{j}\right|^{2}
$$

for $i \neq j$. Changing the roles of $i$ and $j$ and using the symmetry of $\nabla \sigma$ we finally get

$$
\left(b_{i}-b_{j}\right) \operatorname{div} X\left|V_{i}\right|^{2}\left|V_{j}\right|^{2}=0 .
$$

Since $i \neq j$ we get that $\operatorname{div} X=0$ and then $X$ is a parallel field on $M_{0}$.

Now we see that $b_{i}$ are constant on each connected component of $M_{0}$. Taking in Equation (1.1) $V=V_{i}$ and $W=V_{j}$ with $i \neq j$ we obtain

$$
\left\langle(\nabla \sigma)\left(V_{i}, X, X\right), J V_{j}\right\rangle=0 .
$$

Derivating $\left\langle\sigma\left(X, V_{i}\right), J V_{j}\right\rangle=0$ with respect to $X$ and using the above equation and (1.3) we obtain

$$
\left(b_{i}-b_{j}\right)\left\langle\nabla_{X} V_{i}, V_{j}\right\rangle=0,
$$

and so $\nabla_{X} V_{i}$ is a field on $\mathcal{D}_{i}$. Now if we derivate $\sigma\left(X, V_{i}\right)=b_{i} J V_{i}$ with respect to $X$, and use the above information and the parallelism of $X$ in (1.1), we obtain that $X\left(b_{i}\right)=0$, and therefore $b_{i}$ are constant on each component of $M_{0}$. So, by continuity, $A_{J X}$ on $\mathcal{D}$ has also two eigenvalues on the clousure of $M_{0}$, and we get that $M_{0}$ is a closed subset of $M^{\prime}$. This proves the claim.

As $M^{\prime}$ is also connected, there are only two possibilities: Either $M_{0}=M^{\prime}$ or $M_{0}=\emptyset$.

If $M_{0}=M^{\prime}$, then the parallel field $X$ has no zeros and $M^{\prime}=M$. As $\rho=b_{1}+b_{2}$ then $\rho$ is also constant and we prove (A). 
If $M_{0}=\emptyset$, then for any point $p$ of $M^{\prime}, A_{J X}$ has only one eigenvalue $b(p)$ on $\mathcal{D}(p)$, which is constant on the leaves of $\mathcal{D}$.

Given any field $V$ on $\mathcal{D}$, we have that $\sigma(X, V)=b J V$. Then derivating with respect to $X$ and using that $\nabla_{X} V$ is a field on $\mathcal{D}$ we obtain

$$
(\nabla \sigma)(X, X, V)+\frac{\operatorname{div} X}{n} b J V=X(b) J V .
$$

But from (1.1) we have that

$$
(\nabla \sigma)(V, X, X)=(\rho-2 b) \frac{\operatorname{div} X}{n} J V
$$

which, joint to the above equation, gives

$$
X(b)=(\rho-b) \frac{\operatorname{div} X}{n} .
$$

If $h: M^{\prime} \longrightarrow \mathbb{R}$ is the function defined by

$$
h=\frac{c}{4}|X|^{2}+\left(\frac{\operatorname{div} X}{n}\right)^{2}+b^{2}
$$

then using Lemma 1, (ii), (1.5), the fact that $b$ is constant on the leaves of $\mathcal{D}$ and (1.2), we have

$$
\nabla h=\frac{-2 \operatorname{div} X}{n|X|^{2}}\left((-c / 4)|X|^{2}+\frac{\operatorname{Ric}(X)}{n-1}+b^{2}-\rho b\right) X=0 .
$$

So $h$ is a constant $\lambda$. This finishes the proof.

\section{Pseudoumbilical Lagrangian submanifolds of complex space forms.}

In the following result we characterize pseudoumbilical Lagrangian immersions in complex space forms (see Definition 1).

Theorem 1. Let $\phi: M^{n} \longrightarrow \bar{M}^{n}(c)$ be a Lagrangian immersion of a connected manifold $M$ and $\exp : T \bar{M} \longrightarrow \bar{M}$ the exponential map of $\bar{M}$.

(i) $\phi$ is elliptic pseudoumbilical if and only if there exist a point $C \in \bar{M}, a$ vector field (without zeros) $X$ on $M$ and a nontrivial smooth function $h: M \longrightarrow \mathbb{C}$ satisfying $\frac{c}{4}|X|^{2}+|h|^{2}=1$, such that for any point outside the zeros of $h, p \in M-\mathcal{Z}(h)$, the geodesic

$$
\beta_{p}(s)=\exp \left(\phi(p), s f(p) h(p) X_{p}\right)
$$


with

$$
f(p)=\left\{\begin{array}{cl}
1 & \text { when } c=0 \\
\frac{\arccos |h|}{|h X|}(p) & \text { when } c=4 \\
\frac{\cosh ^{-1}|h|}{|h X|}(p) & \text { when } c=-4
\end{array}\right.
$$

pass through the point $C$ at $s=1$. This point $C$ of $\bar{M}$ will be called the center of $\phi$.

(ii) $\phi: M \longrightarrow \mathbb{C H}^{n}$ is hyperbolic pseudoumbilical if and only if there exist a complex hyperplane $\mathbb{C} \mathbb{H}^{n-1}$, a vector field $X$ on $M$ and a nontrivial smooth function $h: M \longrightarrow \mathbb{C}$ satisfying $|h|^{2}-|X|^{2}=-1$, such that for any point outside the zeros of $h, p \in M-\mathcal{Z}(h)$, the geodesic

$$
\beta_{p}(s)=\exp \left(\phi(p), s f(p) h(p) X_{p}\right)
$$

with $f(p)=\frac{\cosh ^{-1}|X|}{|h X|}(p)$ cuts orthogonally to $\mathbb{C H} \mathbb{H}^{n-1}$ at $s=1$.

(iii) $\phi: M \longrightarrow \mathbb{C H}^{n}$ is parabolic pseudoumbilical if and only if there exist a vector field (without zeros) $X$ on $M$ and a smooth function $h: M \longrightarrow$ $\mathbb{C}$ satisfying $|h|^{2}-|X|^{2}=0$ such that the map

$$
p \in M \mapsto\left[\beta_{p}\right] \in \mathbb{S}^{2 n-1}(\infty),
$$

with $\beta_{p}$ the geodesic ray $\beta_{p}(s)=\exp \left(\phi(p), s h(p) X_{p}\right), s \geq 0$, is a constant $C \in \mathbb{S}^{2 n-1}(\infty)$ and the horosphere with infinity point $C$, where $\phi(p)$ lies in, has radius $\delta\left|X_{p}\right|$, with $\delta$ a positive real number.

Remark 3. If $\phi: M \longrightarrow \bar{M}^{n}(c)$ is an elliptic pseudoumbilical Lagrangian immersion and $d$ is the distance on $\bar{M}^{n}(c)$, then

$$
d(C, \phi)=\left\{\begin{array}{cl}
|X| & \text { when } c=0 \\
\arccos |h| & \text { when } c=4 \\
\cosh ^{-1}|h| & \text { when } c=-4 .
\end{array}\right.
$$

So, if $N$ is the leaf of the foliation $\mathcal{D}$ passing through a point $p \in M-\mathcal{Z}(h)$, then $\phi(N)$ lies on the geodesic sphere of $\bar{M}^{n}(c)$ centered at $C$ and radius $d(C, \phi(p))$.

When $c=0$ or $c=-4, \mathcal{Z}(h)=\emptyset$. When $c=4, h$ can have zeros. By continuity, $\mathcal{Z}(h)$ is given by

$$
\mathcal{Z}(h)=\{p \in M: d(\phi(p), C)=\pi / 2\},
$$

i.e., $\phi(\mathcal{Z}(h))$ is the intersection of $\phi(M)$ with the cut locus of the point $C$. 
Also, if $\bar{M}^{n}=\mathbb{C}^{n}, \phi$ is given by $\phi=C+h X$ with $|h|=1$. In this case, if $G: \mathbb{C}^{n}-\{C\} \longrightarrow \mathbb{C}^{n}-\{C\}$ is the inversion centered at the point $C$, then $G \circ \phi$ is also an elliptic pseudoumbilical Lagrangian immersion with center $C$. The corresponding closed and conformal field is $X /|X|^{2}$.

Remark 4. If $\phi: M \longrightarrow \mathbb{C H}^{n}$ is a hyperbolic pseudoumbilical Lagrangian immersion and $d$ is the distance on $\mathbb{C} \mathbb{H}^{n}$, then

$$
d\left(\mathbb{C} \mathbb{H}^{n-1}, \phi\right)=\cosh ^{-1}|X| .
$$

So, if $N$ is the leaf of the foliation $\mathcal{D}$ passing through a point $p \in M$, then $d\left(\mathbb{C H} \mathbb{H}^{n-1}, \phi(N)\right)=\cosh ^{-1}\left|X_{p}\right|$, which means that $\phi(N)$ lies on the tube over $\mathbb{C} \mathbb{H}^{n-1}$ of radius $\cosh ^{-1}\left|X_{p}\right|$. Also

$$
\mathcal{Z}(h)=\left\{p \in M: \phi(p) \in \mathbb{C} \mathbb{H}^{n-1}\right\}
$$

Finally, if $\phi: M \longrightarrow \mathbb{C} \mathbb{H}^{n}$ is a parabolic pseudoumbilical Lagrangian immersion, and $N$ the leaf of the foliation $\mathcal{D}$ passing through a point $p \in M$, then $\phi(N)$ lies on the (2n-1)-dimensional horosphere of $\mathbb{C} \mathbb{H}^{n}$ with infinity point $C$ and radius $\delta\left|X_{p}\right|$.

These $\eta$-umbilic hypersurfaces of $\mathbb{C} \mathbb{H}^{n}$ carry a contact structure and the leaves of the foliation $\mathcal{D}$ of our pseudoumbilical Lagrangian submanifolds are integral submanifolds of maximal dimension of such structure (see $[\mathbf{B}]$ for details).

Proof. Let $\phi: M \longrightarrow \bar{M}^{n}(c)$ a Lagrangian immersion, $h: M \longrightarrow \mathbb{C}$ a nontrivial smooth function, $X$ a vector field without zeros on $M$ and $f$ : $M-\mathcal{Z}(h) \longrightarrow \mathbb{R}$ a smooth function. For each $p \in M-\mathcal{Z}(h), \beta_{p}(s)$ will denote the geodesic $\exp \left(\phi(p), s f(p) h(p) X_{p}\right)$.

Given a vector $v$ tangent to $M$ at $p$ and a curve $\alpha:(-\epsilon, \epsilon) \rightarrow M-\mathcal{Z}(h)$ with $\alpha(0)=p$ and $\alpha^{\prime}(0)=v$, we consider a variation $G:(-\epsilon, \epsilon) \times \mathbb{R} \longrightarrow \bar{M}$ of the geodesic $\beta_{p}$ given by $G(t, s)=\gamma_{t}(s)$, where $\gamma_{t}$ is the geodesic on $\bar{M}$ with $\gamma_{t}(0)=\phi(\alpha(t))$ and $\gamma_{t}^{\prime}(0)=(f h X)(\alpha(t))$. Then $K(s)=\frac{\partial G}{\partial t}(0, s)$ is a Jacobi field along the geodesic $\beta_{p}$.

To determine $K(s)$ we need to control its initial conditions $K(0)$ and $K^{\prime}(0)$. It is clear that $K(0)=\phi_{*}(v) \equiv v$. Also

$$
K^{\prime}(0)=\frac{\partial^{2} G}{\partial s \partial t}(0,0)=\left.\frac{\partial}{\partial t}\right|_{0} \frac{\partial G}{\partial s}(t, 0)=d(f h)_{p}(v) X_{p}+(f h)(p) \bar{\nabla}_{v} X
$$

where $\bar{\nabla}$ is the Levi-Civita connection on $\bar{M}$. 
As $\beta_{p}^{\prime}(0)=(f h X)(p)$, we decompose $K(0)$ and $K^{\prime}(0)$ in the following way

$$
\begin{aligned}
K(0)= & \frac{\mathcal{R}(h)}{f|h X|^{2}}\left\langle X_{p}, v\right\rangle \beta_{p}^{\prime}(0)-\frac{\mathcal{I}(h)}{f|h X|^{2}}\left\langle X_{p}, v\right\rangle J \beta_{p}^{\prime}(0)+v^{\perp}, \\
K^{\prime}(0)= & \langle\nabla \log f|h X|, v\rangle \beta_{p}^{\prime}(0) \\
& +\left(\frac{\left\langle\sigma\left(X_{p}, X_{p}\right), J v\right\rangle}{|X|^{2}}+\frac{\mathcal{I}(v(h) \bar{h})}{|h|^{2}}\right) J \beta_{p}^{\prime}(0)+f h\left(\bar{\nabla}_{v} X\right)^{\perp}
\end{aligned}
$$

where ${ }^{\perp}$ means the component orthogonal to the complex plane spanned by $X_{p}, \mathcal{R}$ (resp. $\mathcal{I}$ ) denotes real (resp. imaginary) part, and $\nabla$ is the gradient of the induced metric. Now, taking into account (2.1), our Jacobi field $K$ along $\beta_{p}$ is given by

$$
K(s)=(\nu s+\mu) \beta_{p}^{\prime}(s)+K_{1}(s)+\hat{K}(s),
$$

being

$$
\nu=\langle\nabla \log f|h X|, v\rangle, \quad \mu=\frac{\Re(h)\langle v, X\rangle}{f|h X|^{2}}
$$

and $K_{1}(s)$ and $\hat{K}(s)$ the Jacobi fields along $\beta_{p}$ given by

$$
\begin{gathered}
K_{1}(s)=\left\{\begin{array}{cc}
A_{1}(s)+s B_{1}(s) & \text { when } c=0 \\
\cos (2 \sqrt{\lambda} s) A_{1}(s)+\frac{\sin (2 \sqrt{\lambda} s)}{2 \sqrt{\lambda}} B_{1}(s) & \text { when } c=4 \\
\cosh (2 \sqrt{-\lambda} s) A_{1}(s)+\frac{\sinh (2 \sqrt{-\lambda} s)}{2 \sqrt{-\lambda}} B_{1}(s) & \text { when } c=-4, \\
\hat{A}(s)+s \hat{B}(s) & \text { when } c=0
\end{array}\right. \\
\hat{K}(s)=\left\{\begin{array}{cc}
\cos (\sqrt{\lambda} s) \hat{A}(s)+\frac{\sin (\sqrt{\lambda} s)}{\sqrt{\lambda}} \hat{B}(s) & \text { when } c=4 \\
\cosh (\sqrt{-\lambda} s) \hat{A}(s)+\frac{\sinh (\sqrt{-\lambda} s)}{\sqrt{-\lambda}} \hat{B}(s) & \text { when } c=-4,
\end{array}\right.
\end{gathered}
$$

where $\lambda=\frac{c}{4}(f|h X|)^{2}(p)$ and $A_{1}(s), B_{1}(s), \hat{A}(s), \hat{B}(s)$ are, respectively, the parallel fields along $\beta_{p}(s)$ with

$$
\begin{aligned}
A_{1}(0) & =-\frac{\mathcal{I}(h)}{f|h X|^{2}}\left\langle X_{p}, v\right\rangle J \beta_{p}^{\prime}(0), \\
B_{1}(0) & =\left(\frac{\left\langle\sigma\left(X_{p}, X_{p}\right), J v\right\rangle}{|X|^{2}}+\frac{\mathcal{I}(v(h) \bar{h})}{|h|^{2}}\right) J \beta_{p}^{\prime}(0), \\
\hat{A}(0) & =v^{\perp}, \quad \hat{B}(0)=f h\left(\bar{\nabla}_{v} X\right)^{\perp} .
\end{aligned}
$$


We remark that $K_{1}(s)$ is a Jacobi field colinear with $J \beta_{p}^{\prime}(s)$ and $\hat{K}(s)$ is a Jacobi field orthogonal to $\beta_{p}^{\prime}(s)$ and $J \beta_{p}^{\prime}(s)$.

Proof of (i). First, we suppose that $\phi$ is elliptic pseudoumbilical. Then (see Definition 1 and Proposition 1$)$ there exists a closed and conformal vector field $X$ (without zeros) such that $\sigma(X, X)=\rho J X$ and $\sigma(X, V)=b J V$ for any field $V$ orthogonal to $X$. Defining $h$ by

$$
h=-\frac{\operatorname{div} X}{n}+i b,
$$

$X$ can be chosen (see Remark 1) in such a way that $|h|^{2}+\frac{c}{4}|X|^{2}=1$. If $h \equiv 0$, then $X$ is a parallel vector field and hence $\operatorname{Ric}(X)=0$. As $b$ also vanishes, (1.2) says that $c=0$, which contradicts the fact that $|h|^{2}+\frac{c}{4}|X|^{2}=1$. So $h$ is nontrivial. In fact the set of points where $h$ does not vanish is dense in $M$.

Now we consider the function $F: M-\mathcal{Z}(h) \longrightarrow \bar{M}$ given by

$$
F(p)=\beta_{p}(1)=\exp \left(\phi(p), f(p) h(p) X_{p}\right) .
$$

We are going to compute $d F_{p}(v)$ for any $p \in M-\mathcal{Z}(h)$ and any $v \in T_{p} M$. It is clear that $d F_{p}(v)=K(1)$, being $K(s)$ the Jacobi field associated to $v$ (see the beginning of the proof).

From the definition of $f(p)$ and the fact that $|h|^{2}=1-\frac{c}{4}|X|^{2}$ we can directly get

$$
\begin{array}{r}
\sin \sqrt{\lambda}=|X|, \quad \cos \sqrt{\lambda}=|h|, \quad \text { when } c=4 \\
\sinh \sqrt{-\lambda}=|X|, \quad \cosh \sqrt{-\lambda}=|h|, \quad \text { when } c=-4 .
\end{array}
$$

From Lemma 1, Proposition 1, (1.2), (1.5) and the expression of $f$, we get that $\mu+\nu=0$. Also, from Lemma 1, Proposition 1, (1.5) and an easy computation, it follows that

$$
B_{1}(0)=b\left(\frac{1}{|X|^{2}}-\frac{c}{4|h|^{2}}\right)\left\langle X_{p}, v\right\rangle J \beta_{p}^{\prime}(0),
$$

which allows to prove, using $(2.2)$, that $K_{1}(1)=0$.

Finally, from Lemma 1 we have that $\hat{B}(0)=-f|h|^{2} v^{\perp}$, which implies using again $(2.2)$ that $\hat{K}(1)=0$. So we have got that

$$
d F_{p}(v)=K(1)=0,
$$

for any tangent vector $v \in T_{p} M$ and any $p \in M-\mathcal{Z}(h)$.

If $c=0$ or $c=-4, \mathcal{Z}(h)=\emptyset$ and then $F: M \longrightarrow \bar{M}$ is a constant function $C$.

If $c=4$, let $M_{1}$ and $M_{2}$ be two different connected components of $M$ $\mathcal{Z}(h)$ with $\bar{M}_{1} \cap \bar{M}_{2} \neq \emptyset$. Then $F$ is constant on each $M_{i}$, i.e., $F\left(M_{i}\right)=C_{i}$ for $i=1,2$. Let $p \in \mathcal{Z}(h) \cap \overline{M_{1}} \cap \overline{M_{2}}$. From Lemma 1, (v) we can parameterize $M$ around $p$ like $(-\delta, \delta) \times N^{n-1}$ such that $\phi\left(0, x_{0}\right)=p$. In this 
neighborhood, $h$ is a function of $t \in(-\delta, \delta)$ (see Lemma 1 again), and so $\{0\} \times N \subset \mathcal{Z}(h)$. So

$$
\phi_{0}: N \longrightarrow \mathbb{C P}^{n}
$$

defined by $\phi_{0}(x)=\phi(0, x)$ is a Lagrangian immersion lying in the cut locus of $C_{1}$ and $C_{2}$. If $C_{1} \neq C_{2}$, then the image of $\phi_{0}$ lies in the intersection of both, which is a linear $(n-2)$-subspace of $\mathbb{C P}^{n}$. This is impossible because $\phi_{0}$ is Lagrangian and $N$ is $(n-1)$-dimensional. So we have obtained that $C_{1}=C_{2}=C$ and the necessary condition is proved.

Conversely, suppose that there exist a tangent vector field $X$ (without zeros) and a nontrivial function $h: M \longrightarrow \mathbb{C}$ such that $F(p)=\beta_{p}(1)=$ $\exp \left(\phi(p), f(p) h(p) X_{p}\right): M-\mathcal{Z}(h) \longrightarrow \bar{M}$ is a constant function. Then $d F_{p}(v)=0$ for any $p \in M-\mathcal{Z}(h)$ and for any $v \in T_{p} M$. So the corresponding Jacobi field $K(s)$ along the geodesic $\beta_{p}$ satisfies $K(1)=d F_{p}(v)=0$. Looking at the expression of $K(s)$, this means that

$$
\mu+\nu=0, \quad K_{1}(1)=0 \quad \text { and } \quad \hat{K}(1)=0 .
$$

But $\mu+\nu=0$ implies, taking into account the expression of $f$, that

$$
\nabla|X|^{2}=-2 \mathcal{R}(h) X .
$$

Also, from $\hat{K}(1)=0$ and using (2.2), we obtain that

$$
|h|^{2} v^{\perp}+h\left(\bar{\nabla}_{v} X\right)^{\perp}=0 .
$$

Now by decomposing the above equation in tangential and normal components to $\phi$ we get

$$
\left(\nabla_{v} X\right)^{\perp}=-\mathcal{R}(h) v^{\perp}, \quad \sigma\left(X_{p}, v\right)^{\perp}=\mathcal{I}(h) J v^{\perp} .
$$

Now (2.3) and the first part of Equation (2.4) say that

$$
\nabla_{v} X=-\mathcal{R}(h) v
$$

for any $v \in T_{p} M$, and for any $p \in M-\mathcal{Z}(h)$. This means that $X$ is a closed and conformal vector field on $M-\mathcal{Z}(h)$ with $\operatorname{div}(X)=-n \mathcal{R}(h)$.

Also, taking $v=X_{p}$ in the second part of Equation (2.4) we obtain that $\sigma(X, X)=\rho J X$ for certain function $\rho$. Finally, taking $v$ orthogonal to $X_{p}$ in the same Equation (2.4), we get $\sigma\left(v, X_{p}\right)=\mathcal{I}(h) J v$. So (see Proposition 1) $\phi: M-\mathcal{Z}(h) \longrightarrow \bar{M}$ is an elliptic pseudoumbilical Lagrangian immersion.

If $c=0,-4$ the proof is finished. If $c=4, \phi(\mathcal{Z}(h))$ is contained in the cut locus of the center $C$, which is a linear $(n-1)$-subspace of $\mathbb{C P}^{n}$. As $\phi$ is a Lagrangian immersion, $\mathcal{Z}(h)$ has no interior points and $M-\mathcal{Z}(h)$ is dense on $M$. So the whole $\phi$ is an elliptic pseudoumbilical immersion.

Proof of (ii). First, we suppose that $\phi: M \longrightarrow \mathbb{C} \mathbb{H}^{n}$ is hyperbolic pseudoumbilical. Then, from Proposition 1 and Definition 1, there exists a closed 
and conformal vector field $X$ (without zeros) such that $\sigma(X, X)=\rho J X$ and $\sigma(X, V)=b J V$ for any $V$ orthogonal to $X$. Defining $h$ by

$$
h=-\frac{\operatorname{div} X}{n}+i b,
$$

$X$ can be chosen (see Remark 1) in such a way that $|h|^{2}-|X|^{2}=-1$. If $h \equiv 0$, then $X$ is parallel and so $\operatorname{Ric}(X)=0$. As $b=0,(1.2)$ gives a contradiction. So $h$ is nontrivial.

In this case, we consider $F: M-\mathcal{Z}(h) \longrightarrow \mathbb{C H}^{n}$ given by

$$
F(p)=\beta_{p}(1)=\exp \left(\phi(p), f(p) h(p) X_{p}\right) .
$$

Following the proof of (i), we can prove in this case that $\mu+\nu=0$ and $K_{1}(1)=0$ and hence

$$
d F_{p}(v)=\hat{K}(1),
$$

which means that $d F_{p}(v)$ is orthogonal to the complex plane spanned by $\beta_{p}^{\prime}(1)$. Also, using the expressions of $\hat{K}(s)$ and $f$, it is easy to check that $\hat{K}^{\prime}(1)=0$, and then $\left\{\beta_{p}^{\prime}(1): p \in M-\mathcal{Z}(h)\right\}$ spans a 1-complex dimensional parallel subbundle on $\mathbb{C H}^{n}$ along $M-\mathcal{Z}(h)$. So, for each connected component $C_{i}$ of $M-\mathcal{Z}(h)$ there exists a complex hyperplane $\mathbb{C} \mathbb{H}_{i}^{n-1}$ of $\mathbb{C H}^{n}$ such that $F\left(C_{i}\right) \subset \mathbb{C H}_{i}^{n-1}$. It is clear that for any $p \in C_{i}, \beta_{p}(s)$ cuts orthogonally to $\mathbb{C H}_{i}^{n-1}$ at $s=1$.

Following a similar reasoning as in (i), we can prove that $M-\mathcal{Z}(h)$ is connected and the proof of the necessary condition is finished.

Conversely, suppose that there exist a vector field $X$ without zeros on $M$, a nontrivial smooth function $h: M \longrightarrow \mathbb{C}$ satisfying $-|X|^{2}+|h|^{2}=-1$ and a linear hyperplane $\mathbb{C} \mathbb{H}^{n-1}$ such that the geodesic $\beta_{p}(s)=\exp (\phi(p), f|h X|(p))$ cuts orthogonally to $\mathbb{C} \mathbb{H}^{n-1}$ at $s=1$. We follow again the proof of (i), and then, if $K$ is the corresponding Jacobi field along $\beta_{p}, d F_{p}(v)=K(1)$ is tangent to $\mathbb{C} \mathbb{H}^{n-1}$. Also, $\beta_{p}^{\prime}(1)$ spans the normal space to $\mathbb{C} \mathbb{H}^{n-1}$ and hence $K^{\prime}(1)$ is a normal vector to $\mathbb{C} \mathbb{H}^{n-1}$ at $\beta_{p}(1)$. This means that $\hat{K}^{\prime}(1)=0$. From these two facts, we obtain using the expression of $K(s)$ that

$$
\mu+\nu=0, \quad K_{1}(1)=0, \quad \hat{K}^{\prime}(1)=0 .
$$

Using the above like in the proof of (i), we can prove that $X$ is a closed and conformal vector field such that $\sigma(X, X)=\rho X$ and $\sigma(X, V)=\mathcal{I}(h) J V$ for any $v$ orthogonal to $X$. So $\phi: M-\mathcal{Z}(h) \longrightarrow \mathbb{C H}^{n}$ is a hyperbolic pseudoumbilical Lagrangian immersion. By continuity, $\phi(\mathcal{Z}(h)) \subset \mathbb{C H}^{n-1}$, and since $\phi$ is Lagrangian, $\mathcal{Z}(h)$ has no interior points, and $M-\mathcal{Z}(h)$ is dense in $M$. This means that the whole $\phi$ is hyperbolic pseudoumbilical.

Proof of (iii). We denote by $F: M \longrightarrow \mathbb{S}^{2 n-1}(\infty)$ the map $F(p)=\left[\beta_{p}\right]$. Let $\widetilde{\phi}$ be a local horizontal lift of $\phi$ to $\mathbb{H}_{1}^{2 n+1}$. Using the notation of Paragraph 1 , 
$\beta_{p}=\Pi\left(\widetilde{\beta_{p}}\right)$, with $\widetilde{\beta}_{p}(s)=\cosh \left(s\left|X_{p}\right|^{2}\right) \widetilde{\phi}(p)+\sinh \left(s\left|X_{p}\right|^{2}\right) h(p) X_{p} /\left|X_{p}\right|^{2}$, where we have identified $X$ and $\widetilde{\phi}_{*} X$. From the identification $\mathbb{S}^{2 n-1}(\infty) \equiv$ $\pi(\mathcal{N})$, we have that

$$
F \equiv \pi \circ \varphi, \text { with } \varphi=\widetilde{\phi}+\frac{h X}{|X|^{2}} .
$$

Suppose now that $\phi$ is parabolic pseudoumbilical. Then, from Proposition 1 and Definition 1 there exists a closed and conformal vector field $X$ (without zeros) such that $\sigma(X, X)=\rho J X$ and $\sigma(X, V)=b J V$ for any $V$ orthogonal to $X$. We define

$$
h=-\frac{\operatorname{div} X}{n}+i b
$$

Then Proposition 1 and Remark 1 say that we can take $X$ in order to $|h|^{2}-|X|^{2}=0$. For any tangent vector $v \in T_{p} M$, using Lemma 1, (1.2) and (1.5), we obtain $v(\varphi)=\frac{h}{|X|^{2}}\langle v, X\rangle \varphi$. This means that $d F_{p}(v)=0$ and from the connection of $M$ this implies that $F$ is a constant function $C \in \mathbb{S}^{2 n-1}(\infty)$. Following the notation of Paragraph 1 , we can take $\widetilde{C}=\varphi$. Using once more Lemma 1, (1.2) and (1.5), it is straightforward to prove that

$$
v\left(\frac{|(\widetilde{\phi}, \widetilde{C})|^{2}}{|X|^{2}\left|\widetilde{C}_{n+1}\right|^{2}}\right)=0 .
$$

This finishes the proof of the necessary condition.

Conversely, if $v$ is any tangent vector to $M$, then $d F_{p}(v)=0$, which implies that

$$
v(\varphi)=\frac{h}{|X|^{2}}\langle v, X\rangle \varphi
$$

On the other hand, since $\phi(p)$ lies in the horosphere with infinity point $C$ and radius $\delta\left|X_{p}\right|$, we deduce that $v\left(\left|\varphi_{n+1}\right|^{2}|X|^{2}\right)=0$. Using (2.5) in this equation we get $\nabla|X|^{2}=-2 \mathcal{R}(h) X$. Using this information in (2.5) and following a similar reasoning like in the proof of (i), we arrive at $\phi$ is parabolic pseudoumbilical.

Theorem 1 shows that elliptic pseudoumbilical Lagrangian immersions have a center and that they can be constructed using the exponential map of the ambient space. This fact will imply that they are invariant under certain transformations of the ambient space, what allows us construct them from the classification given by A. Ros and the third author for complex Euclidean space.

Proposition 2. (i) Let $\phi: M^{n} \longrightarrow \mathbb{C}^{n}$ be an immersion, $\langle$,$\rangle the Eu-$ clidean metric on $\mathbb{C}^{n}$ and $g$ the Fubini-Study metric on $\mathbb{C}^{n}$. Then $\phi$ is 
pseudoumbilical Lagrangian with center 0 in $\left(\mathbb{C}^{n}, g\right)$ if and only if $\phi$ is elliptic pseudoumbilical Lagrangian with center 0 in $\left(\mathbb{C}^{n},\langle\rangle,\right)$.

(ii) Let $\phi: M^{n} \longrightarrow \mathbb{B}^{n} \subset \mathbb{C}^{n}$ be an immersion and $g$ the Bergmann metric on $\mathbb{B}^{n}$. Then $\phi$ is elliptic pseudoumbilical Lagrangian with center 0 in $\left(\mathbb{B}^{n}, g\right)$ if and only if $\phi$ is elliptic pseudoumbilical Lagrangian with center 0 in $\left(\mathbb{B}^{n},\langle\rangle,\right)$.

Remark 5. Proposition 2 is not true when the center of the immersion is a point $C \in \mathbb{C}^{n}$ different from 0 .

Proof of (i). From the definition of $g$, if $\Omega^{g}$ and $\Omega$ denote the Kaehler two-forms on $\left(\mathbb{C}^{n}, g\right)$ and $\left(\mathbb{C}^{n},\langle\rangle,\right)$ respectively, then

$$
\phi^{*} \Omega^{g}=\frac{1}{1+|p|^{2}}\left\{\phi^{*} \Omega-\frac{\phi^{*}(\Im(\alpha \wedge \bar{\alpha}))}{1+|p|^{2}}\right\} .
$$

Also, the expressions of the exponential maps at $0 \in \mathbb{C}^{n}$ with respect to the metrics $\langle$,$\rangle and g$ are given respectively by

$$
\exp (0, v)=v, \quad \exp ^{g}(0, v)=\cos (|v|) \sin (|v|) \frac{v}{|v|},
$$

and it is not difficult to see that the parallel transport along the geodesic $\exp ^{g}(0, t v)$ is (like in the Euclidean case) the identity.

First, we suppose that $\phi$ is an elliptic pseudoumbilical Lagrangian immersion with respect to $\left(\mathbb{C}^{n},\langle\rangle,\right)$. From Theorem 1 we have that

$$
\phi=h X,
$$

with $|h|^{2}=1$.

Then, $\left(\phi^{*} \alpha\right)(v)=\bar{h}\left\langle v, X_{p}\right\rangle$. So from (2.6) we have that $\phi^{*} \Omega^{g}=0$, which means that $\phi$ is a Lagrangian immersion in $\left(\mathbb{C}^{n}, g\right)$.

On the other hand, our immersion $\phi=h X$ can be rewritten as

$$
\phi(p)=\exp ^{g}(0, f(p)(\hat{h} \hat{X})(p)),
$$

with $\hat{h}=\frac{h}{\sqrt{1+|X|^{2}}}, \hat{X}=\sqrt{1+|X|^{2}} X$ and $f(p)$ the corresponding function given in Theorem 1. Although $\left(\mathbb{C}^{n}, g\right)$ is not complete, it is easy to check that Theorem 1 can be used here in order to say that $\phi$ is a pseudoumbilical Lagrangian immersion in $\left(\mathbb{C}^{n}, g\right)$ with $\mathcal{Z}(h)=\emptyset$.

Conversely, we suppose that $\phi$ is a pseudoumbilical Lagrangian immersion with respect to $\left(\mathbb{C}^{n}, g\right)$. From Theorem 1 and the fact that the cut locus of 0 in $\mathbb{C}^{n}$ is empty we have that

$$
\phi=\exp ^{g}(0, f h X),
$$

with $g(X, X)+|h|^{2}=1, f=\frac{\arccos |h|}{|h| \sqrt{g(X, X)}}$ and $\mathcal{Z}(h)=\emptyset$. So using the expression of $\exp ^{g}(0,-)$ we have that

$$
\phi=h X .
$$


So $\left(\phi^{*} \alpha\right)(v)=\frac{\bar{h}}{\sqrt{g(X, X)}}\left\langle v, X_{p}\right\rangle$ and from $(2.6)$ we have that $\phi^{*} \Omega=0$, which means that $\phi$ is a Lagrangian immersion in $\left(\mathbb{C}^{n},\langle\rangle,\right)$.

Now, our immersion $\phi$ can be rewritten as $\phi=\exp (0, \hat{h} \hat{X})$ with $\hat{h}=\frac{h}{|h|}$ and $\hat{X}=|h| X$. This means, using Theorem 1 again, that $\phi$ is an elliptic pseudoumbilical Lagrangian immersion in $\left(\mathbb{C}^{n},\langle\rangle,\right)$. This finishes the proof of (i).

To prove (ii) we follow the proof of (i) taking into account that in this case

$$
\exp ^{g}(0, v)=\cosh (|v|) \sinh (|v|) \frac{v}{|v|},
$$

and the parallel transport along the geodesic $\exp ^{g}(0, t v)$ is the identity.

Proposition 2 allows us, using the results got by A. Ros and the third author for the case of complex Euclidean space, to classify the elliptic pseudoumbilical Lagrangian submanifolds of $\mathbb{C P}^{n}$ and $\mathbb{C} \mathbb{H}^{n}$. In fact, we start recalling the classification for complex Euclidean space.

Theorem $([\mathbf{R U}])$. Let $\phi: M^{n} \longrightarrow \mathbb{C}^{n}$ be an elliptic pseudoumbilical Lagrangian immersion with center $C$. Then locally $M^{n}$ is a product $I \times N$, where $I$ is an interval of $\mathbb{R}$ and $N$ a simply-connected manifold, and $\phi$ is given by

$$
\phi(t, x)-C=\alpha(t) \widetilde{\psi}(x),
$$

where $\widetilde{\psi}: N \longrightarrow \mathbb{S}^{2 n-1} \subset \mathbb{C}^{n}$ is a horizontal lift to $\mathbb{S}^{2 n-1}$ of a Lagrangian immersion $\psi: N \longrightarrow \mathbb{C P}^{n-1}$ and $\alpha: I \longrightarrow \mathbb{C}^{*}$ a regular curve.

The proof of the statements given in the following families of examples is straightforward and so will be omitted.

Examples 1. Let $\alpha: I \longrightarrow \mathbb{C P}^{1}-\{\Pi(0,1)\}$ be a regular curve, $\psi: N \longrightarrow$ $\mathbb{C P}^{n-1}$ a Lagrangian immersion of an (n-1)-dimensional simply-connected manifold $N$, and $\widetilde{\alpha}=\left(\widetilde{\alpha}_{1}, \widetilde{\alpha}_{2}\right): I \longrightarrow \mathbb{S}^{3} \subset \mathbb{C}^{2}$ and $\widetilde{\psi}: N \longrightarrow \mathbb{S}^{2 n-1} \subset \mathbb{C}^{n}$ horizontal lifts of $\alpha$ and $\psi$ respectively. Then,

$$
\alpha * \psi: I \times N \longrightarrow \mathbb{C P}^{n}
$$

given by

$$
(\alpha * \psi)(t, x)=\Pi\left(\widetilde{\alpha}_{1}(t) \widetilde{\psi}(x) ; \widetilde{\alpha}_{2}(t)\right),
$$

is a pseudoumbilical Lagrangian immersion with center $C=\Pi(0, \ldots, 0,1)$.

Remark 6. Looking at Remark 3, it is clear that this immersion $\alpha * \psi$ verifies that

$$
\mathcal{Z}(h)=\{(t, x) \in I \times N: \alpha(t)=\Pi(1,0)\} .
$$


Examples 2. Let $\alpha: I \longrightarrow \mathbb{C H}^{1}-\{\Pi(0,1)\}$ be a regular curve, $\psi: N \longrightarrow$ $\mathbb{C P}^{n-1}$ a Lagrangian immersion of an $(n-1)$-dimensional simply-connected manifold $N$, and $\widetilde{\alpha}=\left(\widetilde{\alpha}_{1}, \widetilde{\alpha}_{2}\right): I \longrightarrow \mathbb{H}_{1}^{3} \subset \mathbb{C}^{2}$ and $\widetilde{\psi}: N \longrightarrow \mathbb{S}^{2 n-1} \subset \mathbb{C}^{n}$ horizontal lifts of $\alpha$ and $\psi$ respectively. Then,

$$
\alpha * \psi: I \times N \longrightarrow \mathbb{C H}^{n}
$$

given by

$$
(\alpha * \psi)(t, x)=\Pi\left(\widetilde{\alpha}_{1}(t) \widetilde{\psi}(x) ; \widetilde{\alpha}_{2}(t)\right),
$$

is an elliptic pseudoumbilical Lagrangian immersion with center $C=\Pi(0$, $\ldots, 0,1)$.

Remark 7. The immersions $\alpha * \psi$ given in Examples 1 and 2 do not depend on the horizontal lift of the curve $\alpha$ and, up to holomorphic isometries of the ambient space, do not depend on the horizontal lift of $\psi$.

Corollary 1. Let $\phi: M^{n} \longrightarrow \mathbb{C P}^{n}$ (respectively $\phi: M^{n} \longrightarrow \mathbb{C H}^{n}$ ) be an elliptic pseudoumbilical Lagrangian immersion. Then $\phi$ is locally congruent to some of the immersions $\alpha * \psi$ described in Examples 1 (respectively in Examples 2).

Proof. We start by proving the projective case. From Theorem $1, \phi$ has a center $C \in \mathbb{C P}^{n}$, and there is no restriction if we take it as $C=\Pi(0, \ldots, 0,1)$. Let

$$
\mathbb{C P}^{n-1}=\left\{\Pi\left(z_{1}, \ldots, z_{n+1}\right) \in \mathbb{C P}^{n}: z_{n+1}=0\right\}
$$

be the cut locus of the point $C$. Then

$$
F: \mathbb{C}^{n} \longrightarrow \mathbb{C P}^{n}-\mathbb{C P}^{n-1},
$$

given by

$$
F(p)=\Pi\left(\frac{1}{\sqrt{1+|p|^{2}}}(p, 1)\right),
$$

is a diffeomorphism with $F(0)=C$ and $F^{*}\langle\rangle=$,$g , where \langle$,$\rangle (respectively$ g) denotes the Fubini-Study metric on $\mathbb{C P}^{n}$ (respectively on $\mathbb{C}^{n}$ ).

From Remark 2 and Proposition 2 it is easy to see that

$$
F^{-1} \circ \phi: M-B \longrightarrow \mathbb{C}^{n},
$$

with $B=\left\{p \in M: \phi(p) \in \mathbb{C P}^{n-1}\right\}$ is an elliptic pseudoumbilical Lagrangian immersion in $\left(\mathbb{C}^{n},\langle\rangle,\right)$ with center 0 , which means, using Theorem [RU], that locally

$$
F^{-1} \circ \phi=\beta \widetilde{\psi}
$$


with $\beta: I \longrightarrow \mathbb{C}^{*}$ a regular curve and $\widetilde{\psi}$ the horizontal lift to $\mathbb{S}^{2 n-1}$ of a Lagrangian immersion $\psi: N \longrightarrow \mathbb{C P}^{n-1}$, being $N$ an $(n-1)$-dimensional simply-connected manifold. So, $\phi: M-B \longrightarrow \mathbb{C P}^{n}-\mathbb{C P}^{n-1}$ is given by

$$
\phi=\Pi\left(\frac{1}{\sqrt{1+|\beta|^{2}}}(\beta \widetilde{\psi}, 1)\right)=\Pi\left(\widetilde{\alpha} 1 \widetilde{\psi}, \widetilde{\alpha}_{2}\right),
$$

where

$$
\left(\widetilde{\alpha}_{1}, \widetilde{\alpha}_{2}\right)=\frac{e^{i \theta}}{\sqrt{1+|\beta|^{2}}}(\beta, 1)
$$

being

$$
\theta(t)=-\int_{t_{0}}^{t} \frac{\left\langle\beta^{\prime}, J \beta\right\rangle}{1+|\beta|^{2}} d r
$$

with $t_{0}$ a point of the interval $I$. From (2.7) it is easy to see that $\widetilde{\alpha}=\left(\widetilde{\alpha}_{1}, \widetilde{\alpha}_{2}\right)$ is a horizontal curve in $\mathbb{S}^{3}$ and so it is the horizontal lift to $\mathbb{S}^{3}$ of the regular curve $\alpha=\Pi \circ \widetilde{\alpha}$ in $\mathbb{C P}^{1}$. This finishes the proof of the projective case.

To prove the hyperbolic case, we proceed in a similar way taking into account that the center $C$ of $\phi$ can be taken as $C=\Pi(0, \ldots, 0,1)$ and that $F: \mathbb{B}^{n} \longrightarrow \mathbb{C H}^{n}$ given by

$$
F(p)=\Pi\left(\frac{1}{\sqrt{1-|p|^{2}}}(p, 1)\right)
$$

is a diffeomorphism with $F(0)=C$ and $F^{*}\langle\rangle=$,$g , being \langle$,$\rangle (respectively$ $g$ ) the metric of $\mathbb{C H}^{n}$ (respectively the Bergmann metric on $\mathbb{B}^{n}$ ).

To finish the description of these submanifolds in $\mathbb{C H} \mathbb{H}^{n}$, it remains the hyperbolic and parabolic cases. As always, we start describing examples whose assertions will not be proved and will take again into account Remark 7 .

Examples 3. Let $\alpha: I \longrightarrow \mathbb{C H}^{1}$ be a regular curve, $\psi: N \longrightarrow \mathbb{C H}^{n-1}$ a Lagrangian immersion of an $(n-1)$-dimensional simply-connected manifold $N$, and $\widetilde{\alpha}=\left(\widetilde{\alpha}_{1}, \widetilde{\alpha}_{2}\right): I \longrightarrow \mathbb{H}_{1}^{3} \subset \mathbb{C}^{2}$ and $\widetilde{\psi}: N \longrightarrow \mathbb{H}_{1}^{2 n-1} \subset \mathbb{C}^{n}$ horizontal lifts of $\alpha$ and $\psi$ respectively. Then

$$
\alpha * \psi: I \times N \longrightarrow \mathbb{C H}^{n}
$$

given by

$$
(\alpha * \psi)(t, x)=\Pi\left(\widetilde{\alpha}_{1}(t), \widetilde{\alpha}_{2}(t) \widetilde{\psi}(x)\right),
$$

is a hyperbolic pseudoumbilical Lagrangian immersion.

To describe the parabolic pseudoumbilical Lagrangian examples in $\mathbb{C H}^{n}$, we need some previous remarks. 
First, we will consider the null vectors $e_{1}=\frac{1}{2}(0, \ldots, 0,1,-1)$ and $e_{2}=$ $\frac{1}{2}(0, \ldots, 0,1,1)$, in such a way that $\left(e_{1}, e_{2}\right)=1 / 2$ where $($,$) is the Hermitian$ product in $\mathbb{C}^{n+1}$.

Second, if $\psi: N^{n-1} \longrightarrow \mathbb{C}^{n-1}$ is a Lagrangian immersion of a simplyconnected manifold $N$, then the complex 1-form $\alpha$ on $\mathbb{C}^{n-1}$ defined before as

$$
\alpha_{p}(v)=\langle v, p\rangle+i\langle v, J p\rangle
$$

verifies that $\psi^{*} \alpha$ is a closed 1 -form and so there exists a complex function $f^{\psi}: N \longrightarrow \mathbb{C}$ such that $d f^{\psi}=2 \psi^{*} \alpha$.

Examples 4. Let $\alpha: I \longrightarrow \mathbb{C H}^{1}$ be a regular curve, $\psi: N \longrightarrow \mathbb{C}^{n-1}$ a Lagrangian immersion of an $(n-1)$-dimensional simply-connected manifold $N$, and $\widetilde{\alpha}=\widetilde{\alpha}_{1} e_{1}+\widetilde{\alpha}_{2} e_{2}: I \longrightarrow \mathbb{H}_{1}^{3} \subset \mathbb{C}_{1}^{2}$ a horizontal lift of $\alpha$. Then

$$
\alpha * \psi: I \times N \longrightarrow \mathbb{C H}^{n}
$$

given by

$$
(\alpha * \psi)(t, x)=\Pi\left(\widetilde{\alpha}_{1}(t) \psi(x) ; \widetilde{\alpha}_{1}(t) e_{1}+\left(\widetilde{\alpha}_{2}(t)-f^{\psi}(x) \widetilde{\alpha}_{1}(t)\right) e_{2}\right)
$$

with $\Re\left(f^{\psi}\right)=|\psi|^{2}$, is a parabolic pseudoumbilical Lagrangian immersion.

Theorem 2. Let $\phi: M^{n} \longrightarrow \mathbb{C} \mathbb{H}^{n}$ be a pseudoumbilical Lagrangian immersion. If $\phi$ is hyperbolic (respectively parabolic), then $\phi$ is locally congruent to some of the immersions described in Examples 3 (respectively in Examples 4$)$.

Proof. We first consider a horizontal lift $\widetilde{\phi}: U \longrightarrow \mathbb{H}_{1}^{2 n+1}$ of $\phi$, where $U$ can be identified (see Lemma 1, (v)) to $I \times N$, where $I$ is an open interval with $0 \in I$ and $N$ is a simply-connected $(n-1)$-manifold. In addition, following the same notation of the proof of Theorem 1 , we can take $X=(\partial / \partial t, 0)$ and, by identifying $X$ and $\widetilde{\phi}_{*} X$, the second fundamental form $\widetilde{\sigma}$ of $\widetilde{\phi}$ satisfies

$$
\widetilde{\sigma}(X, X)=\rho J X+|X|^{2} \widetilde{\phi}, \quad \widetilde{\sigma}(X, v)=b J v,
$$

for any vector $v$ orthogonal to $X$.

Suppose now $\phi$ is hyperbolic pseudoumbilical. From Theorem 1(ii), up to holomorphic isometries, we can take the complex hyperplane $\mathbb{C} \mathbb{H}^{n-1}$ as

$$
\mathbb{C} \mathbb{H}^{n-1}=\left\{\Pi\left(z_{1}, \ldots, z_{n+1}\right): z_{1}=0\right\} .
$$

The equation of a tube of radius $r$ over $\mathbb{C H}^{n-1}$ is $\left|z_{1}\right|=\sinh r$ (cf. [M]). If $r=\cosh ^{-1}\left|X_{p}\right|$ (see Remark 4), it becomes in

$$
\left|\widetilde{\phi}_{1}\right|^{2}=|X|^{2}-1=|h|^{2} \text {. }
$$

Using Theorem 1 again, the geodesic

$$
\beta_{p}(s)=\Pi\left(\cosh (s f|h X|(p)) \widetilde{\phi}+\sinh (s f|h X|(p)) \frac{(h X)(p)}{|h X|(p)}\right)
$$


with $f(p)=\frac{\cosh ^{-1}\left|X_{p}\right|}{\left|h(p) X_{p}\right|}$ cuts orthogonally to $\mathbb{C} \mathbb{H}^{n-1}$ at $s=1$. In particular, $\beta_{p}(1)=\Pi\left(|X| \widetilde{\phi}+\frac{h X}{|X|}\right) \in \mathbb{C H}^{n-1}$, which means that the first component of $\Upsilon=|X|^{2} \widetilde{\phi}+h X$ is zero.

On the other hand, using (2.8) we obtain that $v\left(h X+|h|^{2} \widetilde{\phi}\right)=0$ and so $\widetilde{\alpha}_{1}=-\left(h X+|h|^{2} \widetilde{\phi}\right)$ is a function of $t$, with $\left|\widetilde{\alpha}_{1}\right|^{2}=|h|^{2}$ and, from (2.8) again, satisfies $\widetilde{\alpha}_{1}^{\prime}=-\frac{|X|^{2}}{h} \widetilde{\alpha}_{1}$.

Since $|X|^{2}-|h|^{2}=1$ and $\langle\Upsilon, \Upsilon\rangle=-|X|^{2}$, from above we can write $\widetilde{\phi}=\widetilde{\alpha}_{1}+\Upsilon$ as

$$
\widetilde{\phi}(t, x)=\left(\widetilde{\alpha}_{1}(t), \Upsilon(t, x)\right) .
$$

Using (2.8), we obtain that $\Upsilon^{\prime}=-\bar{h} \Upsilon$, so that $\alpha_{x}(t)=\Upsilon(t, x)=$ $e^{-\int_{0}^{t} \bar{h}(s) d s} \alpha_{x}(0)$ is a plane curve too. If we now put

$$
\widetilde{\psi}(x)=\frac{\alpha_{x}(0)}{\sqrt{-\left\langle\alpha_{x}(0), \alpha_{x}(0)\right\rangle}},
$$

we have that $\langle\widetilde{\psi}, \widetilde{\psi}\rangle=-1$. From the above definition we can write

$$
\widetilde{\phi}(t, x)=\left(\widetilde{\alpha}_{1}(t), \widetilde{\alpha}_{2}(t) \widetilde{\psi}(x)\right),
$$

with $\widetilde{\alpha}_{2}(t)=|X|(0) e^{-\int_{0}^{t} \bar{h}(s) d s}$.

Since $\widetilde{\phi}$ is horizontal, we deduce easily that $\widetilde{\psi}$ is a horizontal immersion in a certain $\mathbb{H}_{1}^{2 n-1}$ and that $\widetilde{\alpha}=\left(\widetilde{\alpha}_{1}, \widetilde{\alpha}_{2}\right)$ is a horizontal curve in $\mathbb{H}_{1}^{3}$. Thus $\phi: U \longrightarrow \mathbb{C H}^{n}$ is the example $\alpha * \psi$ of Examples 3 with $\alpha=\Pi(\widetilde{\alpha})$ and $\psi=\Pi(\widetilde{\psi})$.

In the parabolic case, from Theorem 1, (iii), we can take up to holomorphic isometries the infinity point $C=\pi(\widetilde{C})$ with $\widetilde{C}=(0, \ldots, 0,1,1)$ and identify, following the notation of Paragraph $1, \widetilde{C}$ with $\varphi=\widetilde{\phi}+\frac{h X}{|X|^{2}}$.

Then (2.8) says that $v(\varphi)=0$, for any vector $v$ orthogonal to $X$. In this way, $\varphi=\varphi(t)$ is a null plane curve satisfying, from (2.8) again, $X(\varphi)=h \varphi$. We now put $\widetilde{\alpha_{1}}=-|X|^{2} \varphi$ and it is easy to check that $\widetilde{\alpha}_{1}^{\prime}=-\bar{h} \widetilde{\alpha_{1}}$.

Now we define

$$
\psi=\frac{1}{\widetilde{\alpha}_{1}}\left(\widetilde{\phi}-\widetilde{\alpha}_{1} e_{1}-2\left(\widetilde{\phi}, e_{1}\right) e_{2}\right)
$$

where $e_{1}=\frac{1}{2}(0, \ldots, 0,1,-1)$ and $e_{2}=\frac{1}{2}(0, \ldots, 0,1,1)$. It is clear that $\left(\psi, e_{1}\right)=0$ and from the properties of $\widetilde{\alpha}_{1}($ or $\varphi)$ and the choosing of $e_{1}$ and $e_{2}$ it is not difficult to get that $\left(\psi, e_{2}\right)=0$ and $X(\psi)=0$.

On the other hand, for any $v$ orthogonal to $X$, we have that $\left(\widetilde{\phi}_{*} v, e_{2}\right)=0$ and this implies

$$
\left(\psi_{*} v, \psi_{*} w\right)=\frac{1}{\left|\widetilde{\alpha_{1}}\right|^{2}}\left(\phi_{*} v, \phi_{*} w\right), \quad\left(\psi_{*} v, \psi\right)=-\frac{\left(\phi_{*} v, e_{1}\right)}{\widetilde{\alpha_{1}}}
$$


Let us take $\widetilde{\alpha}_{2}$ as a solution to $\widetilde{\alpha}_{2}^{\prime}+\bar{h} \widetilde{\alpha}_{2}=-e^{-\int \bar{h}} / h$ and define $f=$ $\frac{\widetilde{\alpha}_{2}-2\left(\widetilde{\phi}, e_{1}\right)}{\widetilde{\alpha}_{1}}$. We compute that $X(f)=0$ and $v(f)=2\left(\psi_{*} v, \psi\right)$.

As a summary, we have shown that $\widetilde{\phi}=\widetilde{\alpha}_{1} \psi+\widetilde{\alpha}_{1} e_{1}+\left(\widetilde{\alpha}_{2}-f \widetilde{\alpha}_{1}\right) e_{2}$ and thus $\phi: U \longrightarrow \mathbb{C H}^{n}$ is the example $\alpha * \psi$ of Examples 4 with $\alpha=\Pi(\widetilde{\alpha})$.

\section{The degenerate case for nonflat complex space forms.}

In this paragraph we will classify the family of Lagrangian submanifolds of $\bar{M}^{n}(c)$ admitting a closed and conformal field $X$ with $\sigma(X, X)=\rho J X$ and satisfying condition (A) of Proposition 1 when $\bar{M}$ is a nonflat complex space form. We will refer to this as the degenerate case. In order to make self contained the paper, we start describing this family when $\bar{M}^{n}(c)$ is the complex Euclidean space $\mathbb{C}^{n}$; this description was obtained by A. Ros and the third author in [RU, Proposition 2].

Proposition $([\mathbf{R U}])$. Let $\phi: M^{n} \longrightarrow \mathbb{C}^{n}$ be Lagrangian immersion of a connected manifold $M$ endowed with a closed and conformal vector field $X$ (without zeros) such that $\sigma(X, X)=\rho J X$. If $\phi$ is degenerate (i.e., condition (A) in Proposition 1 is satisfied) then locally $M^{n}$ is a Riemannian product $M_{1}^{n_{1}} \times M_{2}^{n_{2}}$ with $n_{1} \geq 2, n_{2} \geq 1$, and $\phi$ is the product of two Lagrangian immersions $\phi_{i}: M_{i}^{n_{i}} \longrightarrow \mathbb{C}^{n_{i}}, i=1,2$, being $\phi_{1}$ a spherical immersion (i.e., the image of $\phi_{1}$ lies in a hypersphere of $\left.\mathbb{C}^{n_{1}}\right)$.

The assertions given in the following examples are not proved because they are straightforward.

Examples 5. Let $\phi_{i}:\left(N_{i}, g_{i}\right) \longrightarrow \mathbb{C P}^{n_{i}}, i=1,2$ be Lagrangian immersions of $n_{i}$-dimensional simply-connected manifolds $N_{i}, i=1,2$ and $\widetilde{\phi}_{i}: N_{i} \longrightarrow$ $\mathbb{S}^{2 n_{i}+1}$ horizontal lifts of $\phi_{i}, i=1,2$. Given a real number $\delta \in(0, \pi / 4]$ and being $n=n_{1}+n_{2}+1$,

$$
\phi_{1,2}^{\delta}: \mathbb{R} \times N_{1} \times N_{2} \longrightarrow \mathbb{C P}^{n}
$$

given by

$$
\phi_{1,2}^{\delta}(t, p, q)=\Pi\left(\cos \delta e^{i t \tan \delta} \widetilde{\phi_{1}}(p) ; \sin \delta e^{\left.-i t \cot \delta \widetilde{\phi_{2}}(q)\right),}\right.
$$

is a Lagrangian immersion where $X=\left(\frac{\partial}{\partial t}, 0,0\right)$ is a parallel field on $(\mathbb{R} \times$ $\left.N_{1} \times N_{2}, d t^{2} \times \cos ^{2} \delta g_{1} \times \sin ^{2} \delta g_{2}\right)$ with $\sigma(X, X)=-2 \cot 2 \delta J X$. Moreover $\phi$ is degenerate with $b_{1}=\tan \delta$ and $b_{2}=-\cot \delta$.

Examples 6. Let $\phi_{1}:\left(N_{1}, g_{1}\right) \longrightarrow \mathbb{C P}^{n_{1}}$ and $\phi_{2}:\left(N_{2}, g_{2}\right) \longrightarrow \mathbb{C H}^{n_{2}}$ be Lagrangian immersions of $n_{i}$-dimensional simply-connected manifolds $N_{i}$, $i=1,2$ and $\widetilde{\phi_{1}}: N_{1} \longrightarrow \mathbb{S}^{2 n_{1}+1}$ and $\widetilde{\phi_{2}}: N_{2} \longrightarrow \mathbb{H}_{1}^{2 n_{2}+1}$ horizontal lifts of $\phi_{i}, i=1,2$. Given a positive real number $\delta$ and being $n=n_{1}+n_{2}+1$,

$$
\phi_{1,2}^{\delta}: \mathbb{R} \times N_{1} \times N_{2} \longrightarrow \mathbb{C H}^{n}
$$


given by

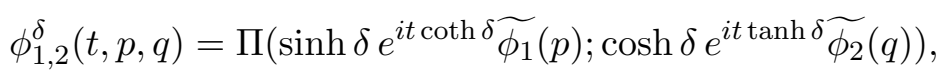

is a Lagrangian immersion where $X=\left(\frac{\partial}{\partial t}, 0,0\right)$ is a parallel field on $(\mathbb{R} \times$ $\left.N_{1} \times N_{2}, d t^{2} \times \sinh ^{2} \delta g_{1} \times \cosh ^{2} \delta g_{2}\right)$ with $\sigma(X, X)=2 \operatorname{coth} 2 \delta J X$. Moreover $\phi$ is degenerate with $b_{1}=\operatorname{coth} \delta$ and $b_{2}=\tanh \delta$.

Proposition 3. Let $\phi: M^{n} \longrightarrow \mathbb{C P}^{n}$ (respectively $\phi: M^{n} \longrightarrow \mathbb{C H}^{n}$ ) be a Lagrangian immersion of a connected manifold $M$ endowed with a closed and conformal vector field $X$ (without zeros) such that $\sigma(X, X)=\rho J X$. If $\phi$ is degenerate, then $\phi$ is locally congruent to some of the immersions $\phi_{1,2}^{\delta}$ described in Examples 5 (respectively in Examples 6).

Proof. We start with the projective case. From Proposition 1, $X$ is parallel and $\rho$ is constant. We assume that $|X|=1$. So the two eigenvalues of $A_{J X}$ on $\mathcal{D}$ (see Proposition 1) satisfy

$$
b_{1}+b_{2}=\rho, \quad b_{1} b_{2}=-1 .
$$

We start taking the distributions $\mathcal{D}_{i}, i=1,2$, on $M$ (see the proof of Proposition 1) defined by

$$
\mathcal{D}_{i}(x)=\left\{v \in \mathcal{D}(x): A_{J X} v=b_{i} v\right\},
$$

of dimensions $n_{1}$ and $n_{2}$ respectively. Using a similar reasoning as in the proof of Proposition 1, it is not difficult to prove that

$$
\nabla_{Z} V_{i} \in \mathcal{D}_{i}
$$

for any $Z$ tangent to $M$ and $V_{i} \in \mathcal{D}_{i}$. So using also Lemma 1, (v), locally $M$ is the Riemannian product $\left(I \times N_{1} \times N_{2}, d t^{2} \times g_{1} \times g_{2}\right)$, where $I$ is an interval of $\mathbb{R}$, and $N_{i}$ are simply-connected manifolds of dimension $n_{i}$.

We consider a horizontal lift $\widetilde{\phi}: I \times N_{1} \times N_{2} \longrightarrow \mathbb{S}^{2 n+1} \subset \mathbb{C}^{n+1}$ of $\phi$, and identify $X$ and $\widetilde{\phi}_{*} X$. If $h$ denotes the second fundamental form of $\widetilde{\phi}$ in $\mathbb{C}^{n+1}$, we have

$$
h(X, X)=\rho J X-\widetilde{\phi}, \quad h\left(X, v_{i}\right)=b_{i} J v_{i},
$$

for any tangent vector $v_{i}$ to $N_{i}$.

We can suppose $b_{1}>0>b_{2}$ and define $\gamma_{i}: I \times N_{1} \times N_{2} \longrightarrow \mathbb{C}^{n+1}, i=1,2$, by

$$
\gamma_{1}=\left(b_{2}-b_{1}\right)\left(b_{2} \widetilde{\phi}+J X\right), \quad \gamma_{2}=\left(b_{1}-b_{2}\right)\left(b_{1} \widetilde{\phi}+J X\right) .
$$

First, it is easy to check that

$$
\left|\gamma_{1}\right|^{2}=b_{2}\left(b_{2}-b_{1}\right)^{3}, \quad\left|\gamma_{2}\right|^{2}=b_{1}\left(b_{1}-b_{2}\right)^{3}, \quad\left\langle\gamma_{1}, \gamma_{2}\right\rangle=\left\langle\gamma_{1}, J \gamma_{2}\right\rangle=0 .
$$

Also, using (3.1) we have

$$
v_{2}\left(\gamma_{1}\right)=0, X\left(\gamma_{1}\right)=b_{1} J \gamma_{1}, v_{1}\left(\gamma_{2}\right)=0, X\left(\gamma_{2}\right)=b_{2} J \gamma_{2},
$$


for any $v_{i}$ tangent to $N_{i}, i=1,2$. So in particular, from (3.2), (3.3) and (3.4), we recuperate $\widetilde{\phi}$ in terms of $\gamma_{i}$ by

$$
\widetilde{\phi}(t, p, q)=\frac{1}{\left(b_{1}-b_{2}\right)^{2}}\left(e^{i b_{1} t} \gamma_{1}(0, p), e^{i b_{2} t} \gamma_{2}(0, q)\right),
$$

for any $(t, p, q) \in I \times N_{1} \times N_{2}$.

Now we define

$$
\begin{gathered}
\widetilde{\phi_{1}}(p)=\frac{\gamma_{1}(0, p)}{\left|\gamma_{1}(0, p)\right|}=\frac{\gamma_{1}(0, p)}{\left(b_{1}-b_{2}\right) \sqrt{b_{2}\left(b_{2}-b_{1}\right)}}, p \in N_{1}, \\
\widetilde{\phi_{2}}(q)=\frac{\gamma_{2}(0, q)}{\left|\gamma_{2}(0, q)\right|}=\frac{\gamma_{2}(0, q)}{\left(b_{1}-b_{2}\right) \sqrt{b_{1}\left(b_{1}-b_{2}\right)}}, q \in N_{2} .
\end{gathered}
$$

So we can rewrite (3.5) as

$$
\widetilde{\phi}(t, p, q)=\left(\sqrt{\frac{b_{2}}{b_{2}-b_{1}}} e^{i b_{1} t} \widetilde{\phi_{1}}(p), \sqrt{\frac{b_{1}}{b_{1}-b_{2}}} e^{i b_{2} t} \widetilde{\phi_{2}}(q)\right) .
$$

Now the properties of the lift $\widetilde{\phi}$ say that $\widetilde{\phi}_{i}$ are horizontal immersions in the corresponding $\mathbb{S}^{2 n_{i}+1} \subset \mathbb{C}^{n_{i}+1}$, which means that $\widetilde{\phi}_{i}$ are horizontal lifts of Lagrangian immersions $\phi_{i}=\Pi\left(\widetilde{\phi}_{i}\right), i=1,2$, in $\mathbb{C P}^{n_{i}}$.

Because $b_{1}=\tan \delta$ for some $\delta \in\left(0, \frac{\pi}{2}\right)$, if $\delta \in\left(0, \frac{\pi}{4}\right]$, our immersion is locally congruent to some of the immersions $\phi_{1,2}^{\delta}$ described in Examples 5 . If $\delta \in\left(\frac{\pi}{\frac{\pi}{\pi}}, \frac{\pi}{2}\right)$, then our immersion is locally congruent to some of the immersions $\phi_{1,2}^{\frac{\pi}{2}-\delta}$ described in Examples 5 .

We follow with the hyperbolic case. As the proof is quite similar to the above proof, we will omit some details. From Proposition 1, $X$ is parallel and $\rho$ is constant. We assume that $|X|=1$. So the two eigenvalues of $A_{J X}$ on $\mathcal{D}$ (see Proposition 1) satisfied

$$
b_{1}+b_{2}=\rho, \quad b_{1} b_{2}=1 .
$$

Reasoning as before, locally $M$ is the Riemannian product $\left(I \times N_{1} \times\right.$ $\left.N_{2}, d t^{2} \times g_{1} \times g_{2}\right)$, where $I$ is an interval of $\mathbb{R}$, and $N_{i}$ are simply-connected manifolds of dimension $n_{i}$.

We consider a horizontal lift $\widetilde{\phi}: I \times N_{1} \times N_{2} \longrightarrow \mathbb{H}_{1}^{2 n+1} \subset \mathbb{C}^{n+1}$, and identify $X$ and $\widetilde{\phi}_{*} X$. If $h$ denotes the second fundamental form of $\widetilde{\phi}$ in $\mathbb{C}^{n+1}$ we have

$$
h(X, X)=\rho J X+\widetilde{\phi}, \quad h\left(X, v_{i}\right)=b_{i} J v_{i},
$$

for any tangent vector $v_{i}$ to $N_{i}$.

We can suppose $b_{1}>b_{2}>0$. Otherwise $0>b_{1}>b_{2}$ and changing $X$ by $-X$ we will be in the first case. We define $\gamma_{i}: I \times N_{1} \times N_{2} \longrightarrow \mathbb{C}^{n+1}$, $i=1,2$, by

$$
\gamma_{1}=\left(b_{2}-b_{1}\right)\left(b_{2} \tilde{\phi}+J X\right), \quad \gamma_{2}=\left(b_{1}-b_{2}\right)\left(b_{1} \tilde{\phi}+J X\right) .
$$


Following the same reasoning as in the projective case, we get that $\widetilde{\phi}$ can be written as

$$
\widetilde{\phi}(t, p, q)=\left(\sqrt{\frac{b_{2}}{b_{1}-b_{2}}} e^{i b_{1} t} \widetilde{\phi_{1}}(p), \sqrt{\frac{b_{1}}{b_{1}-b_{2}}} e^{i b_{2} t} \widetilde{\phi_{2}}(q)\right) .
$$

Now the properties of the lift $\widetilde{\phi}$ say that $\widetilde{\phi}_{i}$ are horizontal immersions in the corresponding $\mathbb{S}^{2 n_{1}+1}$ and $\mathbb{H}_{1}^{n_{2}+1}$, and then $\phi_{1}=\Pi\left(\widetilde{\phi}_{1}\right)$ and $\phi_{2}=\Pi\left(\widetilde{\phi}_{2}\right)$ are Lagrangian immersions in $\mathbb{C P}^{n_{1}}$ and $\mathbb{C} \mathbb{H}^{n_{2}}$ respectively, with horizontal lifts $\widetilde{\phi}_{1}$ and $\widetilde{\phi}_{2}$. As $b_{1} \in(1, \infty)$, let $\delta$ be the positive real number with $\operatorname{coth} \delta=$ $b_{1}$. Then our immersion is locally congruent to some of $\phi_{1,2}^{\delta}$ described in Examples 6.

\section{Lagrangian submanifolds with conformal Maslov form in nonflat complex space forms.}

In this section, we will deal with the case that $X=J H$. Since $J H$ is a closed vector field (because the ambient manifold is a complex space form), we must only assume that $J H$ is a conformal vector field on the submanifold. It is well-known that, up to a constant, $J H$ is the dual vector field of the Maslov form and so this kind of Lagrangian submanifolds will be referred from now on as Lagrangian submanifolds with conformal Maslov form. They were deeply studied by A. Ros and the third author in complex Euclidean space (cf. [RU]).

In the following, we will describe a special family of Lagrangian submanifolds with conformal Maslov form in nonflat complex space forms in terms of (i) minimal Lagrangian immersions in complex projective, complex hyperbolic and complex Euclidean spaces of less dimension and (ii) a two parameter family of curves of $\mathbb{C P}^{1}$ and $\mathbb{C H} \mathbb{H}^{1}$.

To introduce this last family, we start to consider the following 2-parameter $(\lambda, \mu \neq 0)$ family of o.d.e.s:

$$
u^{\prime 2}+\frac{c}{4} e^{2 u}+\left(\frac{n \mu}{n+2} e^{2 u}+\lambda e^{-n u}\right)^{2}=\left\{\begin{array}{ll}
1, & \text { if } c=4 \\
A \in\{1,0,-1\}, & \text { if } c=-4
\end{array} .\right.
$$

We put $h=-u^{\prime}-i\left(\frac{n \mu}{n+2} e^{2 u}+\lambda e^{-n u}\right)$ and for the solution to (4.1) satisfying $u^{\prime}(0)=0$, following the notation of Examples 1-4, we define the curve $\alpha_{\lambda, \mu}$ in $\mathbb{C P}^{1}$ if $c=4$, in $\mathbb{C H}^{1}$ in the other cases, by means of $\alpha_{\lambda, \mu}=\Pi \circ \widetilde{\alpha}_{\lambda, \mu}$ 
where

$$
\begin{aligned}
& \widetilde{\alpha}_{\lambda, \mu}(t)=\left(e^{u(0)} e^{-\int_{0}^{t} \bar{h}(s) d s},|h(0)| e^{\frac{c}{4} \int_{0}^{t} \frac{e^{2 u(s)}}{h(s)} d s}\right), \text { if } c=4 \text { or } c=-4, A=1, \\
& \widetilde{\alpha}_{\lambda, \mu}(t)=\left(|h(0)| e^{-\int_{0}^{t} \frac{e^{2 u(s)}}{h(s)} d s}, e^{u(0)} e^{-\int_{0}^{t} \bar{h}(s) d s}\right), \text { if } c=-4, A=-1, \\
& \widetilde{\alpha}_{\lambda, \mu}(t)=e^{-\int_{0}^{t} \bar{h}(s) d s}\left(2 e^{2 u(0)} e_{1}-\left(e^{-2 u(0)} / 2+\int_{0}^{t} d s / h(s)\right) e_{2}\right), \\
& \text { if } c=-4, A=0 .
\end{aligned}
$$

Corollary 2. Let $\phi: M^{n} \longrightarrow \bar{M}^{n}(c)$ be a nonminimal Lagrangian immersion. Then $\phi$ has conformal Maslov form and $J H$ is a principal direction of $A_{H}$ if and only if around each point where $H$ does not vanish, $\phi$ is congruent to:

(a) Some $\alpha * \psi$ of Examples 1-4, where $\psi$ is a minimal immersion in $\mathbb{C P}^{n-1}, \mathbb{C} \mathbb{H}^{n-1}$ or $\mathbb{C}^{n-1}$ and $\alpha=\alpha_{\lambda, \mu}$ is the curve given in (4.2). In this case, $|H|=\mu e^{u}$.

(b) Some $\phi_{1,2}^{\delta}$ of Examples 5 with $\phi_{i}$ minimal immersions in $\mathbb{C P}^{n_{i}}, i=1,2$, $\left(n_{1}+n_{2}+1=n\right)$ and arbitrary $\delta \in(0, \pi / 4]$, or some $\phi_{1,2}^{\delta}$ of Examples 6 with $\phi_{1}$ a minimal immersion in $\mathbb{C P}^{n_{1}}, \phi_{2}$ a minimal immersion in $\mathbb{C H}^{n_{2}},\left(n_{1}+n_{2}+1=n\right)$ and arbitrary $\delta>0$.

Remark 8. In [Ch2], B.Y. Chen introduced the notion of Lagrangian Humbilical submanifolds in Kaehler manifolds. This kind of submanifolds correspond to the simplest Lagrangian submanifolds satisfying the two following conditions: $J H$ is an eigenvector of the shape operator $A_{H}$ and the restriction of $A_{H}$ to the orthogonal subspace to $J H$ is proportional to the identity. He classified them in $\mathbb{C P}^{n}$ and $\mathbb{C H}^{n}$ (cf. [Ch2]) proving that, except in some exceptional cases, they are obtained from Legendre curves in $\mathbb{S}^{3}$ or in $\mathbb{H}_{1}^{3}$ via warped products. In Corollary 2 , they appear in (a) by considering $\psi$ totally geodesic.

Proof. It is a consequence of Proposition 1, Corollary 1, Theorem 2, Proposition 3 and the following facts:

First, when we study the geometric properties of the examples $\alpha * \psi$ we arrive at the following expressions for its mean curvature vector:

$$
\begin{array}{r}
n H^{*}=\frac{1}{\left|\widetilde{\alpha}_{1}\right|^{2}}\left((\rho+(n-1) b) J X+(n-1)\left(\widetilde{\alpha}_{1} H_{\psi}^{*} ; 0\right)\right) \\
\left(\operatorname{resp.} n H^{*}=\frac{1}{\left|\widetilde{\alpha}_{2}\right|^{2}}\left((\rho+(n-1) b) J X+(n-1)\left(0 ; \widetilde{\alpha}_{2} H_{\psi}^{*}\right)\right)\right)
\end{array}
$$


for Examples 1, 2, 4 (resp. for Examples 3), where

$$
\begin{array}{r}
\rho=\frac{\left|\widetilde{\alpha}_{1}\right|}{\left|\widetilde{\alpha}^{\prime}\right|^{3}}\left\langle\widetilde{\alpha}^{\prime \prime}, J \widetilde{\alpha}^{\prime}\right\rangle, \quad b=\frac{\left\langle\widetilde{\alpha}_{1}^{\prime}, J \widetilde{\alpha}_{1}\right\rangle}{\left|\widetilde{\alpha}_{1}\right|\left|\widetilde{\alpha}^{\prime}\right|} \\
\left(\text { resp. } \rho=\frac{\left|\widetilde{\alpha}_{2}\right|}{\left|\widetilde{\alpha}^{\prime}\right|^{3}}\left\langle\widetilde{\alpha}^{\prime \prime}, J \widetilde{\alpha}^{\prime}\right\rangle, \quad b=\frac{\left\langle\widetilde{\alpha}_{2}^{\prime}, J \widetilde{\alpha}_{2}\right\rangle}{\left|\widetilde{\alpha}_{2}\right|\left|\widetilde{\alpha}^{\prime}\right|}\right) .
\end{array}
$$

So if we put $X_{\mu}=\mu X=J H$ (where $X$ is normalized according to Remark 1) and look at the foregoing expressions we deduce that $H_{\psi}^{*}=0$ and hence $\psi$ is a minimal immersion, and using that $|X|^{2}=\left|\widetilde{\alpha}_{1}\right|^{2}$ in Examples 1, 2, 4 (resp. $|X|^{2}=\left|\widetilde{\alpha}_{2}\right|^{2}$ in Examples 3), we obtain

$$
\begin{array}{r}
-\mu n\left|\widetilde{\alpha}_{1}\right|^{2}=\rho+(n-1) b \\
\left(\text { resp. }-\mu n\left|\widetilde{\alpha}_{2}\right|^{2}=\rho+(n-1) b\right)
\end{array}
$$

for Examples 1, 2, 4 (resp. for Examples 3). From (1.5) and the above equations, we deduce

$$
b^{\prime}+n u^{\prime} b=-n \mu u^{\prime} e^{2 u}
$$

where $e^{2 u}=|X|^{2}=|H|^{2} / \mu^{2}$, and then

$$
b=-\left(\frac{n \mu}{n+2} e^{2 u}+\lambda e^{-n u}\right) .
$$

Second, the expressions given for $\widetilde{\alpha}$ in Corollary 2 can be deduced following a constructive reasoning from the proof of Corollary 1 and Theorem 2 in all the cases.

Finally, a similar technique can be used to get the cases given in (b).

Next we will consider the easiest examples provided in Corollary 2, taking $\psi=\psi_{0}$ totally geodesic and the curves $\alpha_{0, \mu}$, i.e., putting $\lambda=0$ in (4.1). For our purposes, it is enough to consider $\mu>0$. We will state along this paragraph that these examples play the role of "umbilical" Lagrangian immersions in non flat complex space forms. If $\lambda=0,(4.1)$ becomes in

$$
u^{\prime 2}+\frac{c}{4} e^{2 u}+\nu^{2} e^{4 u}=\left\{\begin{array}{ll}
1, & \text { if } c=4 \\
A \in\{1,0,-1\}, & \text { if } c=-4
\end{array},\right.
$$

with $\nu=\frac{n \mu}{n+2}>0$, and we must now take into account that $h=-u^{\prime}-i \nu e^{2 u}$ for the curves given in (4.2). The solution to (4.3) satisfying $u^{\prime}(0)=0$ is 
given by

$$
e^{2 u(t)}=\left\{\begin{array}{cl}
\frac{2}{\sqrt{1+4 \nu^{2}} \cosh (2 t)+c / 4}, & \text { if } c=4 \text { or } c=-4, A=1, \\
\frac{2}{1-\sqrt{1-4 \nu^{2}} \cos (2 t)}, & \text { if } c=-4, A=-1, \\
\frac{1}{\nu^{2}+t^{2}}, & \text { if } c=-4, A=0 .
\end{array}\right.
$$

The only constant solution appears in the hyperbolic case $c=-4, A=-1$, just when $\nu=1 / 2$. In this case $e^{2 u(t)} \equiv 2$ and the corresponding immersion has parallel mean curvature vector, just like the examples collected in Corollary 2, (b).

In the elliptic case, we define $\theta=(1 / 2) \cosh ^{-1} \sqrt{1+4 \nu^{2}}>0$ and abbreviate $\operatorname{ch}_{\theta}=\cosh \theta$ and $s h_{\theta}=\sinh \theta$; now (4.3) and its solution are rewritten as

$$
u^{\prime 2}+\frac{c}{4} e^{2 u}+\operatorname{ch}_{\theta}^{2} \operatorname{sh}_{\theta}^{2} e^{4 u}=1
$$

and

$$
e^{2 u(t)}=\frac{2}{c h_{2 \theta} \cosh (2 t)+c / 4} .
$$

From (4.2), after a long straightforward computation, we arrive at

$$
\begin{aligned}
\widetilde{\alpha}_{\theta}(t) & =\frac{1}{c h_{\theta} \cosh t+i s h_{\theta} \sinh t}\left(1, s h_{\theta} \cosh t+i c h_{\theta} \sinh t\right), \\
\widetilde{\alpha}_{\theta}(t) & =\frac{1}{\operatorname{sh}_{\theta} \cosh t+i \operatorname{ch}_{\theta} \sinh t}\left(1, c h_{\theta} \cosh t+i s h_{\theta} \sinh t\right),
\end{aligned}
$$

for $c=4$ and $c=-4$ respectively. By identifying $\mathbb{R}^{n}-\{0\}$ with $\mathbb{R} \times \mathbb{S}^{n-1}$ via the conformal transformation $w \mapsto(\log |w|, w /|w|), \alpha_{\theta} * \psi_{0}$ defines a Lagrangian immersion $\phi_{\theta}: \mathbb{R}^{n}-\{0\} \longrightarrow \mathbb{C P}^{n}$, and it is not complicated to check that $\phi_{\theta}$ extends regularly to 0 and $\infty$; hence via stereographic projection we obtain a family of Lagrangian immersions

$$
\phi_{\theta}: \mathbb{S}^{n} \longrightarrow \mathbb{C P}^{n}, \theta>0
$$

given by

$$
\phi_{\theta}\left(x_{1}, \ldots, x_{n}, x_{n+1}\right)=\left[\left(\frac{\left(x_{1}, \ldots, x_{n}\right)}{\operatorname{ch}_{\theta}+i s h_{\theta} x_{n+1}} ; \frac{\operatorname{sh}_{\theta} \operatorname{ch}_{\theta}\left(1+x_{n+1}^{2}\right)+i x_{n+1}}{\operatorname{ch}_{\theta}^{2}+s h_{\theta}^{2} x_{n+1}^{2}}\right)\right]
$$

that we will call the Whitney spheres of $\mathbb{C P}^{n}$. We notice that $\phi_{\theta}$ are embeddings except in a double point and that if $\theta \rightarrow 0$ it appears the totally geodesic immersion of $\mathbb{S}^{n}$ in $\mathbb{C P}^{n}$. 
In a similar way we obtain the Whitney spheres of $\mathbb{C} \mathbb{H}^{n}$,

$$
\Phi_{\theta}: \mathbb{S}^{n} \longrightarrow \mathbb{C H}^{n}, \theta>0,
$$

given by

$$
\Phi_{\theta}\left(x_{1}, \ldots, x_{n}, x_{n+1}\right)=\left[\left(\frac{\left(x_{1}, \ldots, x_{n}\right)}{s h_{\theta}+i c h_{\theta} x_{n+1}} ; \frac{\operatorname{sh}_{\theta} \operatorname{ch}_{\theta}\left(1+x_{n+1}^{2}\right)-i x_{n+1}}{s h_{\theta}^{2}+c h_{\theta}^{2} x_{n+1}^{2}}\right)\right],
$$

which are also embeddings except in a double point.

In the hyperbolic case, we define $\beta=(1 / 2) \cos ^{-1}\left(\sqrt{1-4 \nu^{2}}\right) \in(0, \pi / 4]$ and abbreviate $c_{\beta}=\cos \beta$ and $s_{\beta}=\sin \beta$; now (4.3) and its solution are rewritten as

$$
u^{\prime 2}-e^{2 u}+s_{\beta}^{2} c_{\beta}^{2} e^{4 u}=-1
$$

and

$$
e^{2 u(t)}=\frac{2}{1-c_{2 \beta} \cos 2 t} .
$$

From (4.2), after a long straightforward computation, we arrive at

$$
\widetilde{\alpha}_{\beta}(t)=\frac{1}{s_{\beta} \cos t+i c_{\beta} \sin t}\left(c_{\beta} \cos t-i s_{\beta} \sin t, 1\right) .
$$

If $\mathbb{R} \mathbb{H}^{n-1}=\left\{y=\left(y_{1}, \ldots, y_{n}\right) \in \mathbb{R}^{n}: y_{1}^{2}+\ldots y_{n-1}^{2}-y_{n}^{2}=-1\right\}$ denotes the $(n-1)$-dimensional real hyperbolic space, $\alpha_{\beta} * \psi_{0}$ defines a family of Lagrangian embeddings

$$
\Psi_{\beta}: \mathbb{S}^{1} \times \mathbb{R} \mathbb{H}^{n-1} \longrightarrow \mathbb{C} \mathbb{H}^{n}, \beta \in(0, \pi / 4],
$$

given by

$$
\Psi_{\beta}\left(e^{i t}, y\right)=\left[\frac{1}{s_{\beta} \cos t+i c_{\beta} \sin t}\left(c_{\beta} \cos t-i s_{\beta} \sin t ; y\right)\right] .
$$

We note that $\Psi_{\pi / 4}\left(e^{i t}, y\right)=\left(e^{-2 i t}, \sqrt{2} e^{-i t} y\right)$ is flat.

Finally, in the parabolic case, using (4.2) a straightforward computation leads to

$$
\widetilde{\alpha}_{\nu}(t)=\frac{1}{\nu+i t}\left(\frac{2}{\nu} e_{1}-\nu\left(\frac{\nu^{2}+t^{2}}{2}+i \nu t\right) e_{2}\right) .
$$

From Examples $4, \alpha_{\nu} * \psi_{0}$ defines a one-parameter family of Lagrangian embeddings

$$
\varphi_{\nu}: \mathbb{R}^{n} \equiv \mathbb{R} \times \mathbb{R}^{n-1} \longrightarrow \mathbb{C H}^{n}, \nu>0
$$

given by

$$
\varphi_{\nu}(t ; x)=\left[\frac{1}{\nu+i t}\left(\frac{2}{\nu} x ; \frac{2}{\nu} e_{1}-\left(\frac{\nu\left(\nu^{2}+t^{2}\right)}{2}+\frac{2|x|^{2}}{\nu}+i \nu^{2} t\right) e_{2}\right)\right] .
$$


Theorem 3. Let $\phi: M^{n} \longrightarrow \mathbb{C P}^{n}$ (respectively $\phi: M^{n} \longrightarrow \mathbb{C H}^{n}$ ) be a Lagrangian immersion.

(i) The second fundamental form of $\phi$ is given by

$$
\langle\sigma(v, w), J z\rangle=\frac{n}{n+2} \partial_{v, w, z}\langle v, w\rangle\langle H, J z\rangle
$$

for any tangent vectors $v, w$ and $z$, (where the symbol $\partial_{v, w, z}$ means cyclic sum over $v, w, z)$ if and only if either $\phi$ is totally geodesic or $\phi(M)$ is an open set of some of the Lagrangian submanifolds (4.4) in $\mathbb{C P}^{n}$ (respectively of the Lagrangian submanifolds (4.5), (4.6) or (4.7) in $\left.\mathbb{C} \mathbb{H}^{n}\right)$.

(ii) [B.Y. Chen] The scalar curvature $\tau$ of $M$ satisfies

$$
\tau \leq \frac{n^{2}(n-1)}{n+2}|H|^{2}+n(n-1) \frac{c}{4}
$$

and the equality holds if and only if $\phi$ is totally geodesic or $\phi$ is an open set of some of the Lagrangian submanifolds (4.4) in $\mathbb{C P}^{n}$ (respectively of the Lagrangian submanifolds (4.5), (4.6) or (4.7) in $\mathbb{C H}^{n}$ ).

Remark 9. In [CU2] when $n=2$ and in [Ch1] in arbitrary dimension, the sharp inequality of (ii) between the squared mean curvature and the scalar curvature for a Lagrangian submanifold in a nonflat complex space form was established. By utilising Jacobi's elliptic functions and warped products, B.Y. Chen introduced in [Ch1] three families of Lagrangian submanifolds and two exceptional ones characterized by satisfying the equality in (ii) besides the totally geodesic ones. Later, in $[\mathbf{C h V}]$, explicit expressions of these Lagrangian immersions were found in a different context from ours. All of them are exactly the Lagrangian submanifolds described in (4.4), (4.5), (4.6) and (4.7).

Proof. For the proof of (i), it is straightforward to verify that the totally geodesic immersions and the Lagrangian immersions (4.4)-(4.7) satisfy (4.8).

Conversely, exactly the same proof of Theorem 2 in $[\mathbf{R U}]$ works also here to get that $J H$ is a conformal vector field on $M$. If $H \equiv 0$ then (4.8) implies that $\phi$ is totally geodesic. If $H$ is nontrivial, Lemma 1, (i) says that the zeros of $H$ are isolated. Hence the set of points of $M$ where $H$ does not vanish, say $M^{\prime}$, is a connected open dense subset of $M$. We will work in it. From (4.8) we first deduce that $\sigma(J H, J H)=\frac{3 n}{n+2}|H|^{2} H$. Then Corollary 2 says that $\phi$ is locally congruent to some of the examples described there. Second, (4.8) also gives us that $\sigma(v, J H)=-\frac{n}{n+2}|H|^{2} J v$ for $v$ orthogonal to $J H$ and this implies that necessarily that $\phi$ is locally congruent to some $\alpha * \psi$ of Corollary 2, (a) and, in addition, $\lambda=0$ in (4.1). Using again (4.8) we obtain, for $v$ and $w$ orthogonal to $J H$, that $\sigma(v, w)=\frac{n}{n+2}\langle v, w\rangle H$ and this means that $\sigma_{\psi}^{*}=0$ when we study the second fundamental form of such an immersion $\alpha * \psi$. Thus $\psi$ is totally geodesic. As $M^{\prime}$ is connected and 
dense in $M$, a standard argument shows that $\phi(M)$ is an open set in some of the Lagrangian submanifolds (4.4)-(4.7).

Our proof of (ii) follows from (i) and a standard interpolation argument.

Theorem 4. Let $\phi: M^{n} \longrightarrow \mathbb{C P}^{n}$ (respectively $\phi: M^{n} \longrightarrow \mathbb{C H}^{n}$ ) be a Lagrangian immersion of a compact manifold $M$ with conformal Maslov form. If the first Betti number of $M$ vanishes, then $\phi$ is either minimal (necessarily in $\mathbb{C P}^{n}$ ) or congruent to some of the Whitney spheres (4.4) in $\mathbb{C P}^{n}$ (respectively congruent to some of the Whitney spheres (4.5) in $\mathbb{C H}^{n}$ ).

Proof. Suppose (only in the projective case) that $H$ does not vanishes identically. Since $J H$ is a closed vector field and the first Betti number of $M$ is zero, there exists a function $f$ on $M$ such that $J H=\nabla f$. So $H$ has zeros at the critical points of $f$. Hence $J H$ is a closed and conformal vector field with at least a zero. Under these conditions, exactly the same reasoning of Theorem 3 in [RU] proves that $M$ is conformally equivalent to a round sphere, the leaves of the umbilical foliation $\mathcal{D}$ (see Lemma 1 ) are spheres and $Y=J \sigma(J H, J H)-(3 n /(n+2))|H|^{2} J H$ is a harmonic vector field. Since the first Betti number of $M$ is zero, we deduce that $J H$ is an eigenvector of $A_{H}$. If $N$ is one of the leaves of $\mathcal{D}$, using Corollary 2 we have a minimal Lagrangian immersion $\psi$ necessarily in $\mathbb{C P}^{n-1}$ because in $\mathbb{C}^{n-1}$ and $\mathbb{C} \mathbb{H}^{n-1}$ there are no compact minimal submanifolds. Then we can use the same proof in the above mentioned theorem of $[\mathbf{R U}]$ to conclude that $\psi$ is totally geodesic and that $\sigma$ is given as in (4.8) on the whole of $M$. The proof finishes thanks to Theorem 3.

Next, we obtain the following corollary using the same argument that in Corollary 5 of [RU], where the Whitney spheres are characterised in terms of the behaviour of the Ricci curvature.

Corollary 3. Let $\phi: M^{n} \longrightarrow \mathbb{C P}^{n}$ be a Lagrangian nonminimal immersion of a compact manifold $M$ with conformal Maslov form. Then:

(i) $\operatorname{Ric}(J H) \geq(n-1)|H|^{2}$ if and only if $\phi$ is congruent to some of the Whitney spheres (4.4) in $\mathbb{C P}^{n}$;

(ii) $\operatorname{Ric}(J H) \geq 0$ if and only if either $\phi$ has parallel mean curvature vector or $\phi$ is congruent to some of the Whitney spheres (4.4) in $\mathbb{C P}^{n}$.

To finish this section we state that if our Lagrangian submanifold has first Betti number equal to one then it belongs to our family.

Corollary 4. Let $\phi: M^{n} \longrightarrow \mathbb{C P}^{n}$ (respectively $\phi: M^{n} \longrightarrow \mathbb{C H}^{n}$ ) be a Lagrangian immersion of an orientable compact manifold $M$ with nonparallel conformal Maslov form such that the first Betti number of $M$ is one. 
Then the universal covering of $\phi$ is congruent to some $\alpha * \psi$ as described in Corollary 2,(a).

Proof. From the proof of Theorem 4 we can deduce that $H$ has no zeros on $M$ and so the vector field $|H|^{-n} J H$ is well-defined on $M$. Using Lemma 1, one can check that it is a harmonic vector field on $M$ and the same happens for $Y=J \sigma(J H, J H)-(3 n /(n+2))|H|^{2} J H$. By the hypothesis, there is a constant $a \in \mathbb{R}$ such that

$$
\sigma(J H, J H)=\left(\frac{3 n}{n+2}|H|^{2}+a|H|^{-n}\right) H
$$

and we can go to Corollary 2 to get the result.

\section{References}

[B] D.E. Blair, Contact Manifolds in Riemannian Geometry, Springer-Verlag, (1976), MR 57 \#7444, Zbl 319.53026.

[CU1] I. Castro and F. Urbano, Lagrangian surfaces in the complex Euclidean plane with conformal Maslov form, Tôhoku Math. J., 45 (1993), 565-582, MR 94j:53064, Zbl 792.53030 .

[CU2] _ Twistor holomorphic Lagrangian surfaces in the complex projective and hyperbolic planes, Ann. of Glob. Anal. and Geom., 13 (1995), 59-67, MR 96c:53093, Zbl 827.53059.

[Ch1] B.Y. Chen, Jacobi's elliptic functions and Lagrangian immersions, Proc. Royal Soc. Edinburgh, 126 (1996), 687-704, MR 97k:53071, Zbl 866.53045.

[Ch2] - Interaction of Legendre curves and Lagrangian submanifolds, Israel J. Math., 99 (1997), 69-108, MR 98i:53086, Zbl 884.53014.

$[\mathrm{ChV}]$ B.Y. Chen and L. Vrancken, Lagrangian submanifolds satisfying a basic equality, Math. Proc. Cambridge Phil. Soc., 120 (1996), 291-307, MR 97e:53101, Zbl 860.53034.

[HL] R. Harvey and H.B. Lawson, Calibrated geometries, Acta Math., 148 (1982), 47157, MR 85i:53058, Zbl 584.53021.

[K] M. Kon, Pseudo-Einstein real hypersurfaces in complex space forms, J. Differential Geometry, 14 (1979), 339-354, MR 81k:53050, Zbl 461.53031.

[M] S. Montiel, Real hypersurfaces of a complex hyperbolic space, J. Math. Soc. Japan, 37 (1985), 515-535, MR 96i:53027, Zbl 554.53021.

[RU] A. Ros and F. Urbano, Lagrangian submanifolds of $\mathbb{C}^{n}$ with conformal Maslov form and the Whitney sphere, J. Math. Soc. Japan, 50 (1998), 203-226, MR 98k:53081, Zbl 906.53037.

Received September 13, 1999 and revised March 31, 2000. This research was partially supported by a DGICYT grant No. PB97-0785.

Departamento de Matemáticas Escuela Politécnica Superior

UNIVERSIDAD DE JAÉN

23071 JAÉN

SPAIN 
E-mail address: icastro@ujaen.es

E-mail address: crodri@ujaen.es

Departamento de Geometría y Topología

UNIVERSIDAD DE GRANADA

18071 GRANADA

SPAIN

E-mail address: furbano@goliat.ugr.es 